ARTICLE

DOI: $10.1038 / s 41467-018-06365-0$

\title{
NEDDylation promotes nuclear protein aggregation and protects the Ubiquitin Proteasome System upon proteotoxic stress
}

Chantal M. Maghames ${ }^{1}$, Sofia Lobato-Gil ${ }^{1,2}$, Aurelien Perrin ${ }^{1}$, Helene Trauchessec ${ }^{1}$, Manuel S. Rodriguez ${ }^{2}$, Serge Urbach ${ }^{3}$, Philippe Marin ${ }^{3} \&$ Dimitris P. Xirodimas (1) ${ }^{1}$

Spatial management of stress-induced protein aggregation is an integral part of the proteostasis network. Protein modification by the ubiquitin-like molecule NEDD8 increases upon proteotoxic stress and it is characterised by the formation of hybrid NEDD8/ubiquitin conjugates. However, the biological significance of this response is unclear. Combination of quantitative proteomics with biological analysis shows that, during proteotoxic stress, NEDDylation promotes nuclear protein aggregation, including ribosomal proteins as a major group. This correlates with protection of the nuclear Ubiquitin Proteasome System from stress-induced dysfunction. Correspondingly, we show that NEDD8 compromises ubiquitination and prevents targeting and processing of substrates by the proteasome. Moreover, we identify HUWE1 as a key E3-ligase that is specifically required for NEDDylation during proteotoxic stress. The study reveals a specific role for NEDD8 in nuclear protein aggregation upon stress and is consistent with the concept that transient aggregate formation is part of a defence mechanism against proteotoxicity.

${ }^{1}$ CRBM, CNRS, Univ. Montpellier, UMR5237, Montpellier Cedex 5, 34090, France. ${ }^{2}$ ITAV CNRS USR3505, IPBS-UPS, 1 Place Pierre Potier, Oncopole Entrance B, 31106 Toulouse, France. ${ }^{3}$ Institute of Functional Genomics, 141 rue de la Cardonille, 34094 Montpellier Cedex 5, France. Correspondence and requests for materials should be addressed to D.P.X. (email: dimitris.xirodimas@crbm.cnrs.fr) 
M aintaining a proper and functioning proteome is challenging for cells given the variety of proteotoxic stresses that constantly cause protein damage ${ }^{1,2}$. Aberrant proteins tend to form aggregates that have been linked to many neurodegenerative diseases (Alzheimer's disease, Parkinson's disease), cancer and aging ${ }^{3-7}$. To prevent aggregation, a protein quality control (PQC) network ensures protein repair and refolding by molecular chaperones or clearance of terminally damaged proteins by lysosomal and proteasomal degradation ${ }^{8,9}$. While PQC systems in the cytosol have been well-studied ${ }^{8}$, knowledge on the nuclear PQC is still limited, especially in mammalian cells. The Ubiquitin Proteasome System (UPS) is an essential PQC pathway, as it eliminates misfolded proteins prone to aggregation. Degradation by the $26 \mathrm{~S}$ proteasome depends on the covalent modification of substrates with ubiquitin. Upon activation by the E1-activating enzymes UBA1 or UBA6, ubiquitin is transferred to E2-conjugating enzymes before the modification mainly of lysine residues on target proteins through the action of E3 ligases ${ }^{10}$. Upon ubiquitination, proteins are recognised by shuttle factors that ensure substrate delivery to the $26 \mathrm{~S}$ proteasome. Receptors on the $19 \mathrm{~S}$ lid subunit of the proteasome allow recognition of ubiquitinated proteins followed by ATP-dependent protein unfolding and de-ubiquitination, before translocation of the substrate into the catalytic $20 \mathrm{~S}$ core for degradation ${ }^{11}$. Each of the above steps is independently and finely regulated and collectively they define the rate of substrate degradation ${ }^{12}$. Proteasomes exist both in the cytoplasm and nucleus of mammalian cells, but their relative abundance and activity depend on the cell type and growing conditions ${ }^{13}$. Dysfunction of the UPS is often related to the appearance of protein aggregates in several neurodegenerative diseases ${ }^{14-16}$. However, it is not clear whether formation of aggregates is always a cause of UPS toxicity ${ }^{14-17}$ or it can be part of a defence mechanism against proteotoxicity ${ }^{18-21}$.

While ubiquitin is considered as a main effector in the PQC network, members of the ubiquitin-like molecules family are emerging as new and important regulators of protein homoeostasis 22,23 . NEDD8 is the closest homologue to ubiquitin with $60 \%$ sequence identity and it is conjugated to proteins in a three-step process, similarly to ubiquitin. Nevertheless, NEDD8 employs its own specific enzymes, a heterodimer NEDD8 E1activating enzyme (NAE) and NEDD8 E2-conjugating enzymes, $\mathrm{UBE} 2 \mathrm{M}$ (UBC12) and UBE2F $\mathrm{F}^{24,25}$. The NAE-dependent functions of NEDD8 have been well characterised, mainly in homoeostatic conditions, through NEDDylation of the cullin family of proteins and stimulation of the Cullin-Ring-Ligases (CRLs) activity, but also through NEDDylation of non-cullin substrates ${ }^{24,25}$. Early studies showed that in several neurodegenerative disorders NEDD8 colocalises with ubiquitin and proteasome components in protein inclusions, suggesting a role for NEDD8 in protein aggregation ${ }^{26-28}$. More recently, a role for the NEDD8 interacting protein NUB1 was proposed in the regulation of tau aggregation in Alzheimer's disease ${ }^{29}$ and mutant Huntington toxicity ${ }^{30}$. In addition, a direct link between the NEDD8 pathway and proteotoxic stress was also revealed. Exposure of cells to heat shock, proteasome inhibitors or oxidative stress increases protein NEDDylation. However, the stress-induced NEDD8 conjugation depends on the ubiquitin E1-activating enzyme UBA1 and not on NAE. This was quite surprising given the specificity of E1 enzymes in activating their cognate molecules $^{31}$. A key characteristic of the NEDD8 response to stress is the formation of hybrid NEDD8/ubiquitin conjugates ${ }^{32}$. While the above studies indicate a link between NEDD8 and proteotoxicity, the biological significance of the NEDD8 response to proteotoxic stress remains unclear. We report that NEDD8 promotes nuclear protein aggregation during proteotoxic stress and protects the nuclear UPS from stress-induced dysfunction. Mechanistically, NEDD8 compromises substrate ubiquitination and prevents their processing/degradation by the proteasome. Importantly, these effects are independent of NEDD8 activation by NAE and of the CRL function. This study identifies NEDDylation as a regulatory pathway of protein aggregation and UPS function in the nucleus during proteotoxic stress and is consistent with the concept that transient protein aggregation is a defence mechanism against proteotoxicity.

\section{Results}

Characterisation of the NEDD8 response to proteotoxic stress. In addition to pre-existing proteins, newly synthesised polypeptides are particularly vulnerable and damaged under proteotoxic stress $^{33}$. Treatment of cells with the translational inhibitor Cycloheximide (CHX) prevented the increase in protein NEDDylation upon HS, indicating that the NEDD8 response to proteotoxic stress depends on protein synthesis (Fig. 1a). To determine if the stress-induced NEDDylation depends, at least in part, on damage of newly synthesised proteins we used puromycin, a protein synthesis inhibitor known to induce shortening and damage of newly synthesised proteins at low doses $(5-20 \mu \mathrm{g} /$ $\mathrm{ml})$ and to completely block protein synthesis at high doses (100 $\mu \mathrm{g} / \mathrm{ml})^{34}$. Low concentrations of puromycin induced protein NEDDylation in the absence of any additional stress and showed an additive effect on NEDD8 accumulation when combined with HS. In contrast, when used at a high dose, puromycin reduced the NEDD8 response to HS similarly to the CHX treatment (Fig. 1b). Short-term inhibition of HSP70, which is expected to increase the production of misfolded newly synthesised proteins ${ }^{35}$, also induced NEDDylation, consistent with the data obtained with puromycin (Supplementary Figure 1A). Pulse labelling experiments, where newly synthesised ${ }^{35}$ S-labelled NEDD8 was isolated by immunoprecipitation, showed that the increase in NEDDylation upon stress is not due to an increase in NEDD8 synthesis (Supplementary Figure 1B).

To determine the state of stress-induced NEDDylated proteins, we performed a subcellular fractionation in untreated and HS cells, separating soluble (cytosol, nucleoplasm) from insoluble (pellet) fractions. NEDDylated substrates showed a transition from the cytosolic soluble into the insoluble pellet fraction during HS, indicating aggregation of the modified substrates (Fig. 1c). A well-characterised effect of proteotoxic stress is to induce the formation of cytoplasmic protein aggregates known as aggresomes ${ }^{36}$. However, by immunofluorescence analysis we found that NEDD8 accumulated only in the nucleus upon HS and colocalised with ubiquitin (Fig. 1d). Interestingly, when cells were subjected to a severe proteotoxic stress (overnight MG132 treatment), we detected a reorganisation of the nuclear NEDD8/ubiquitin aggregates (Fig. 1e, Supplementary Figure 2A). $3 \mathrm{D}$ reconstruction showed that the observed structures are a sphere and that NEDD8 and ubiquitin colocalise on its surface (Fig. 1e).

Stress-induced NEDDylation is reported to depend on the activation of NEDD8 by the ubiquitin E1 enzyme UBA1 and not by NAE. Indeed, no effect on HS-induced NEDD8/ubiquitinstained nuclear aggregates was observed upon inhibition of NAE with the specific inhibitor MLN4924 (Fig. 1f). In contrast, treatment of cells with the specific UBA1 inhibitor MLN7243 caused a dramatic decrease in NEDD8/ubiquitin-stained aggregates (Fig. 1f). A dramatic decrease in NEDD8 but not ubiquitin staining in nuclear aggregates was observed upon NEDD8 knockdown by siRNA, showing the antibody specificity (Fig. 1f). Similar results were obtained upon severe proteotoxic stress with MG132 (Supplementary Figure 2B). These data suggest that 
a

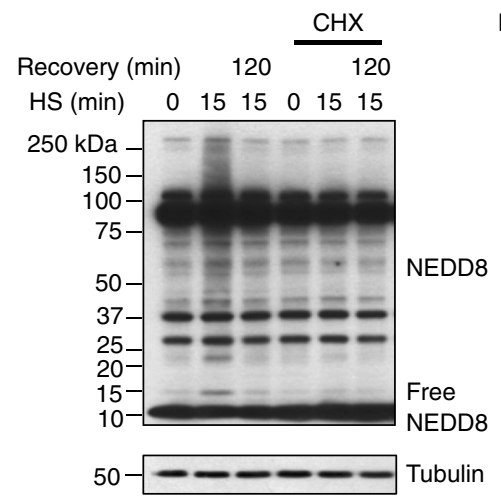

d
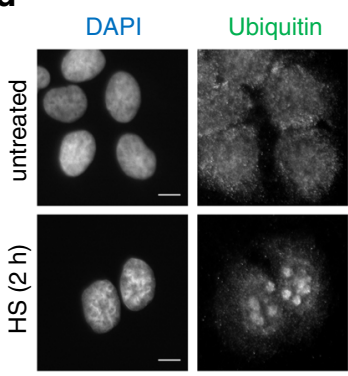

$\mathbf{f}$
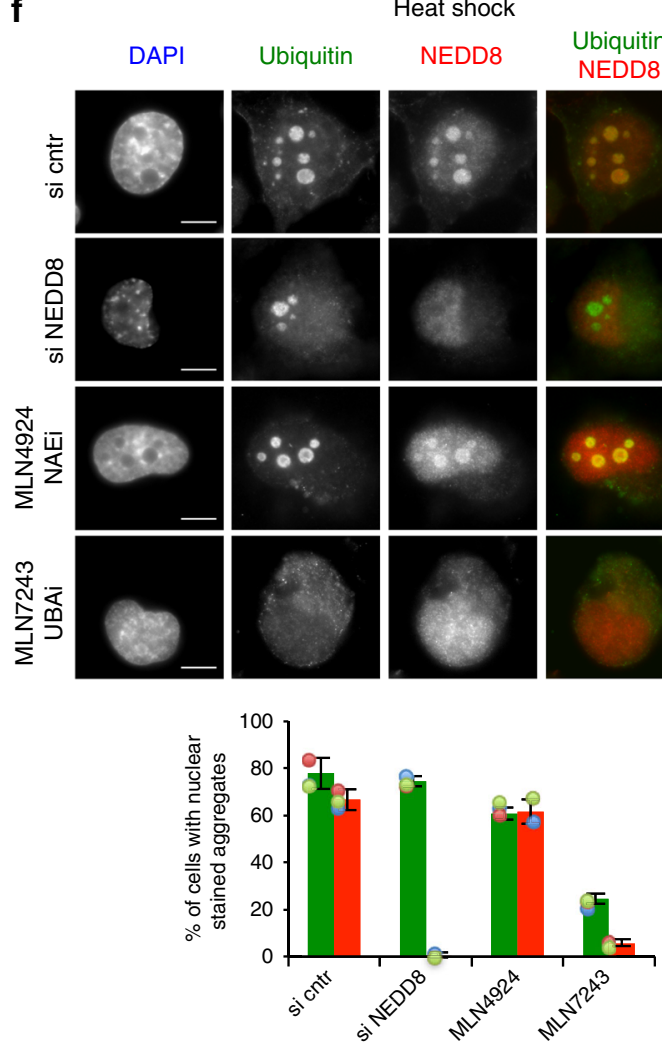

the formation of stress-induced NEDD8 nuclear aggregates depends on the activation of NEDD8 by UBA1. The HSinduced NEDD8/ubiquitin aggregates are transient and disappear when cells are allowed to recover at $37^{\circ} \mathrm{C}$ (Fig. 1g, Supplementary

b

Puromycin $(\mu \mathrm{g} / \mathrm{ml})$

15

10.

35

Ubiquitin
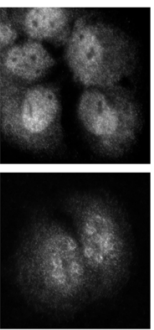

Heat shock
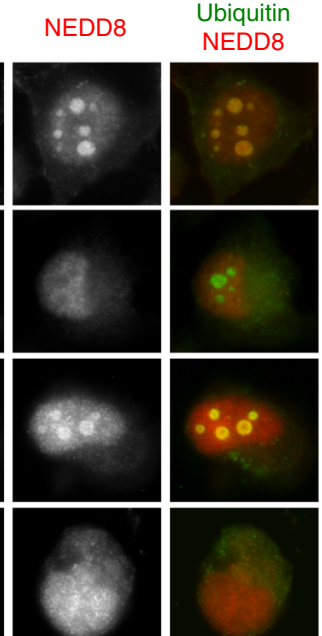

Ubiquitin - NEDD8 $\frac{0.1}{0} \frac{1}{4} \frac{5}{0400}$

HS (min) $040040040040 \quad \overline{40}$

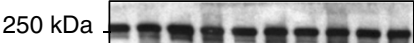

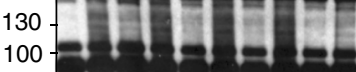

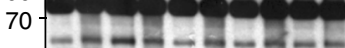

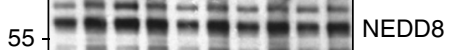

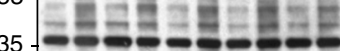
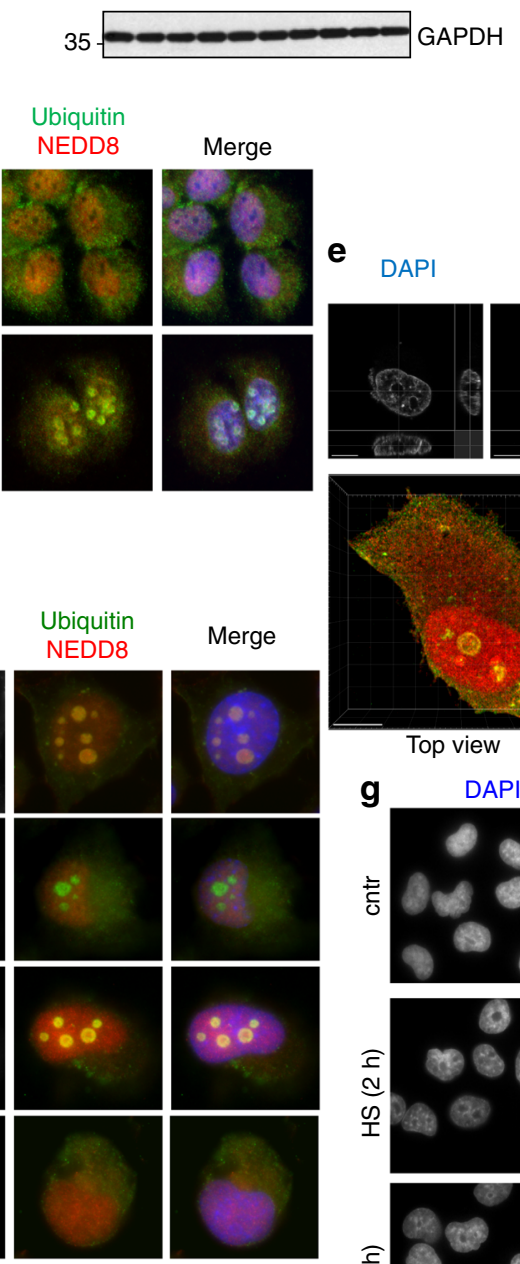

C

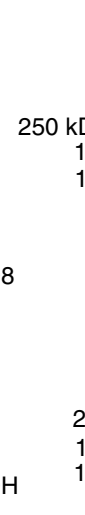

Input

in

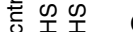

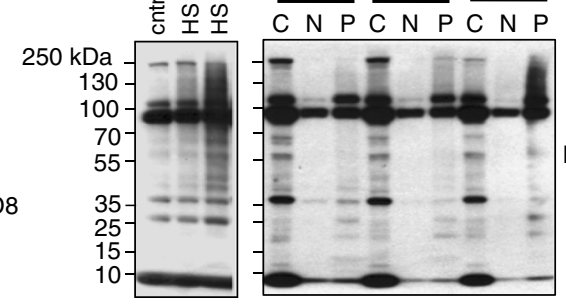

NEDD8
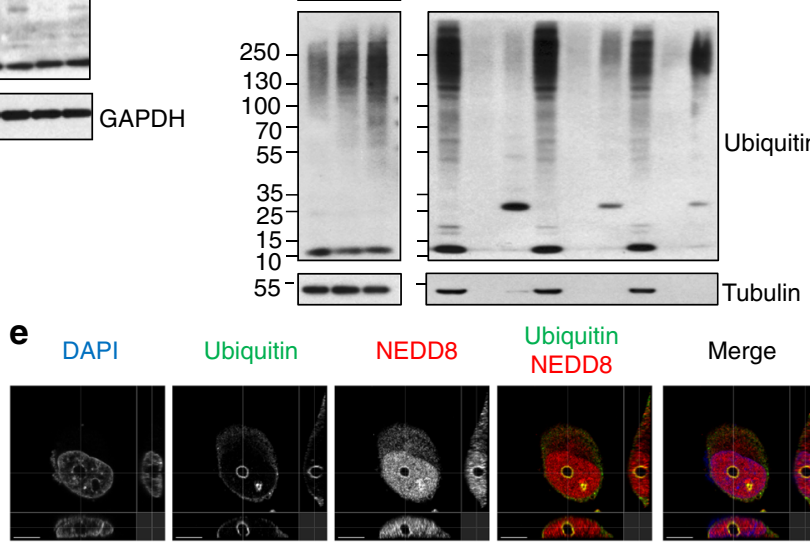

Ubiquitin

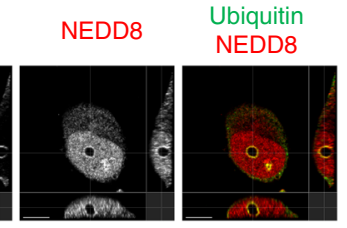

Merge

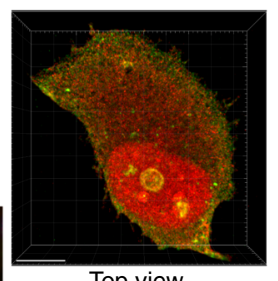

Top view
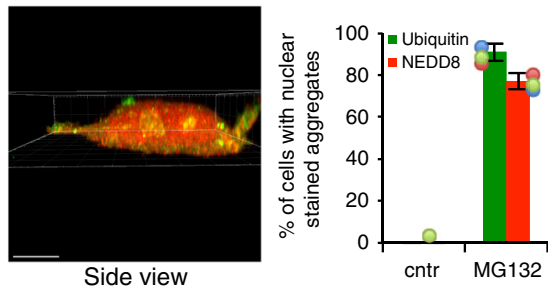

g

DAPI
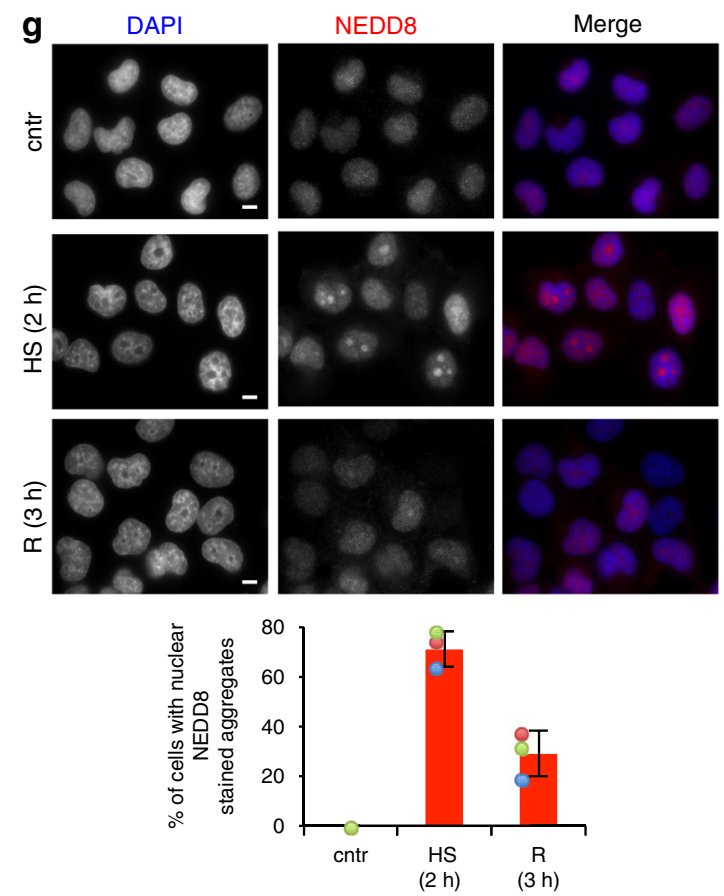

Figure 2C). The combination of the above data indicates that HS induces a progressive accumulation of transient and reversible NEDD8 protein aggregates in the nucleus through NEDD8 activation by UBA1. 
Fig. 1 NEDD8 targets newly synthesised proteins and accumulates in nuclear aggregates upon proteotoxic stress. a U2OS cells were pretreated or not with Cycloheximide (CHX) at $100 \mu \mathrm{g} / \mathrm{ml}$ for $90 \mathrm{~min}$, and then treated as indicated. After heat shock at $43^{\circ} \mathrm{C}$, cells were recovered at $37^{\circ} \mathrm{C}$ for $2 \mathrm{~h}$. $\mathbf{b} \mathrm{U} 2 \mathrm{OS}$ cells were pretreated or not with puromycin at the indicated concentrations for $30 \mathrm{~min}$ then heat-shocked. Cell extracts were analysed by western blotting. c U2OS cells were either untreated or heat-shocked at $43^{\circ} \mathrm{C}$ for the indicated time. After HS, cells were subjected to subcellular fractionation as described in Methods (C, Cytoplasm; N, Nucleoplasm; P, Pellet) and fractions were analysed by western blotting. Tubulin was used as fractionation marker. $\mathbf{d}$ U2OS cells were either untreated or heat-shocked before staining for NEDD8 (red) and ubiquitin (green). Nuclei were stained with DAPI (blue). e Similar as in d, but cells were pretreated with MG132 ( $5 \mu$ M-overnight-ON). 3D rendering was performed as described in Methods. Top panel represents single plane images in different axis. Bottom images are the 3D reconstructions for the top and side view. Scale bar: $10 \mu \mathrm{m}$. Bottom right graph represents the \% of cells with nuclear aggregates stained with NEDD8 or ubiquitin. Approximately 100 cells were counted per condition. f siRNA transfection in H1299 cells was performed as indicated for a total of $36 \mathrm{~h}$. MLN4924 and MLN7243 were used at $500 \mathrm{nM}$ for 15 and $5 \mathrm{~h}$ respectively before heat shock. Staining and quantitation of the experiment (bottom graph) were performed as above. $\mathbf{g ~ H} 1299$ cells were heat-shocked as before and were allowed to recover at $37{ }^{\circ} \mathrm{C}$ as indicated. After fixation, cells were stained for NEDD8 (red) and DAPI (blue). Bottom graph represents quantitation of the experiment in ( $\mathbf{g}$ ), performed as above. For all bar graphs, values represent the average of three independent experiments \pm standard deviation (SD). Scale bars, $10 \mu \mathrm{m}$

NEDDylation promotes nuclear aggregates formation and protects nuclear UPS. Based on the ubiquitin staining, the immunofluorescence analysis indicates that the formation of nuclear aggregates upon proteotoxic stress is not dramatically affected upon NEDD8 knockdown (Fig. 1f, Supplementary Figure $2 \mathrm{~B}$ ). To determine whether instead the composition of nuclear aggregates is controlled by NEDD8, we followed a SILAC mass spectrometry-based proteomic approach. We first determined the composition of protein aggregates induced by HS. U2OS cells were labelled with light (K0R0) or heavy (K8R10) medium and either left at $37^{\circ} \mathrm{C}$ (light) or heat-shocked (heavy) at $43^{\circ} \mathrm{C}$ for $2 \mathrm{~h}$. Cells were mixed 1:1 ratio before subcellular fractionation, isolation of the insoluble pellet fraction and mass spectrometry analysis (Fig. 2a). Peptides for approximately 1500 proteins were quantified in two replicate experiments (Supplementary Data 1). Consistent with the western blot and immunofluorescence analysis, ubiquitin and NEDD8 increased in abundance in the formed insoluble inclusions upon HS (Fig. 2b). Increased abundance was also observed for SUMO-1, 2 in accordance with the established role of SUMOylation in the HS response (Fig. 2b) and the emerging concept for the cross-talk between ubiquitin and Ubls upon stress conditions ${ }^{22,37,38}$. A striking observation was that among proteins with increased abundance in the aggregates, RNA transport and ribosomal proteins (RPs) were the two major groups, accounting for approximately $40 \%$ of the total number (Fig. $2 \mathrm{~b}, \mathrm{c}$ ).

Having determined the composition of the HS-induced aggregates we then assessed the potential role of NEDD8 in the stress-induced nuclear protein aggregation. Based on previous studies $^{39}$, we found that short-term (36-48 h) knockdown of NEDD8, while it fully blocks the stress-induced NEDD8 response, it does not have a major impact on cullin NEDDylation, a key target of the NAE-dependent NEDD8 conjugation (Fig. $2 \mathrm{~d})^{24,25}$. Importantly, under these conditions the levels of well-established targets of CRLs (p21, CDT1) remain unaffected, indicating that the small decrease observed in cullin NEDDylation has no significant effect on the CRL function (Supplementary Figure 3). While these observations suggest that NEDD8 knockdown mainly impacts on stress-induced NEDDylation, defects due to inhibition of NAE-dependent NEDDylation cannot be excluded. To discriminate these effects we performed two SILAC experiments: We determined the effect of NEDD8 knockdown on the HS-induced aggregate composition and in parallel we performed a similar experiment using instead the NAE inhibitor MLN4924 (Fig. 2e, Supplementary Figure 4A, B). As shown (Fig. 2d, Supplementary Figure 3), MLN4924 has no effect on stress-induced NEDDylation but almost completely blocks NAEdependent NEDDylation, including cullin modification and CRL function. We reasoned that the comparison of the two experiments should eliminate any effects due to inhibition of
NAE-dependent NEDDylation and indicate the role of stressinduced NEDDylation on protein aggregation.

Comparison of the two SILAC experiments shows that NEDD8 knockdown affects the composition of the HS-induced aggregates (Fig. 2f, Supplementary Figure 4A, B). Both reduced and increased aggregation was observed (Fig. 2f, g, Supplementary Figure 4C), but clearly the most profound effect of NEDD8 knockdown is to reduce protein aggregation. In particular, a large number (30 IDs) of RPs (Fig. 2f, g) was identified as the most enriched group of proteins with reduced aggregation. Importantly, these effects were specific for NEDD8 knockdown and not observed upon inhibition of the NAE-dependent NEDD8 pathway and CRL function by MLN4924 (Fig. 2f, g, Supplementary Figure 4B). The proteomic analysis indicates that proteotoxic stress-induced NEDDylation mainly promotes aggregation.

The mutual functional relationship between protein aggregation and UPS function is well-established. However, it is still controversial whether stress-induced protein aggregation is detrimental for UPS function or rather a defence mechanism to protect UPS from the increase load of misfolded proteins during stress $^{14-16}$. We therefore determined the role of NEDDylation in UPS function during the HS response. We used HEK293 cells stably expressing GFPu, a fusion of GFP with a peptide that targets GFP for ubiquitin-dependent proteasomal degradation. By monitoring the protein levels of GFPu, the UPS activity can be assessed $^{40}$. We used GFPu constructs fused either to a nuclear export (NES/cytoplasmic) or nuclear import (NLS/nuclear) signal, thus allowing the assessment of the cytoplasmic or nuclear UPS activity ${ }^{18}$. While HS had no effect on NES-GFPu, a progressive increase in NLS-GFPu was observed, indicating that HS specifically compromises the UPS in the nucleus (Fig. 3a, b). This could be due to the reduced UPS activity in the nucleus compared to the cytoplasm or due to the lack of auxiliary proteolytic machineries such as the autophagy pathway ${ }^{13,35}$ Importantly, once stress is alleviated, nuclear UPS is recovered (Fig. 3b). Inhibition of NEDDylation by NEDD8 knockdown further compromised the nuclear UPS activity with no effect on cytoplasmic UPS. Importantly, NEDD8 knockdown reduced UPS activity only during the HS response and not in unstressed conditions. No significant effects on UPS function were observed upon inhibition of the NAE-dependent NEDDylation by MLN4924 (Fig. 3c, d, Supplementary Figure 5A). Monitoring the expression of NLS-GFPu mRNA and rates of protein synthesis shows that the observed effects of NEDD8 knockdown on NLS-GFPu levels are not due to transcriptional/protein synthesis defects (Supplementary Figure 5B, C). Ectopic expression of NEDD8 resistant to siRNA restores the NEDD8 response to stress and rescues the effect of NEDD8 knockdown on nuclear UPS activity (Fig. 3e). The above-described defects in the UPS upon inhibition of NEDDylation could be explained by a 


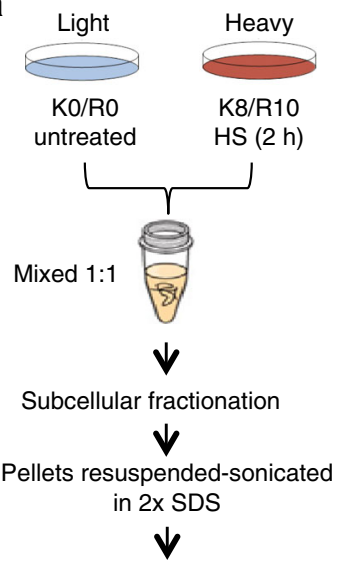

In gel trypsin digestion

$\sqrt{ }$

Peptides extraction and MS analysis/quantitation

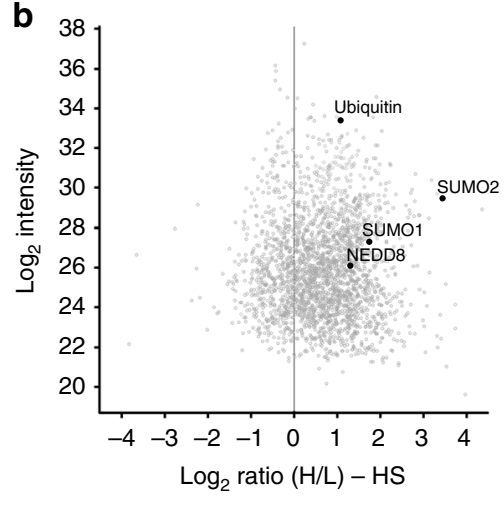

C

\begin{tabular}{lcc}
\hline \multicolumn{3}{c}{ Increased aggregation upon HS } \\
\hline Pathway description & $\begin{array}{c}\text { Gene } \\
\text { count }\end{array}$ & $\begin{array}{c}\text { False } \\
\text { discovery rate }\end{array}$ \\
\hline RNA transport & 45 & $8.23 e-23$ \\
Ribosome & 39 & $2.65 e-20$ \\
\hline $\begin{array}{l}\text { Proteasome } \\
\text { Aminoacyl-tRNA }\end{array}$ & 22 & $5.23 e-17$ \\
biosynthesis & 16 & $1.17 e-09$ \\
$\begin{array}{l}\text { Protein processing in } \\
\text { endoplasmic reticulum }\end{array}$ & 30 & $1.33 e-09$ \\
$\begin{array}{l}\text { Epstein-Barr virus } \\
\text { infection }\end{array}$ & 32 & $2.79 e-09$ \\
\begin{tabular}{l} 
DNA replication \\
\hline
\end{tabular} & 12 & $6.97 e-07$ \\
\hline
\end{tabular}

e

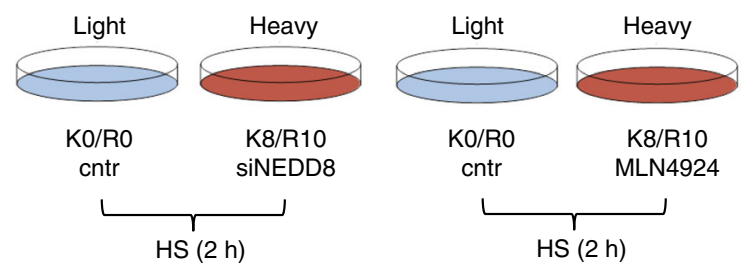

NEDD8

g

\begin{tabular}{|c|c|c|}
\hline \multicolumn{3}{|c|}{ Decreased aggregation (siNEDD8 specific) } \\
\hline Pathway description & $\begin{array}{l}\text { Gene } \\
\text { count }\end{array}$ & $\begin{array}{c}\text { False } \\
\text { discovery rate }\end{array}$ \\
\hline Ribosome & 30 & $1.23 e-22$ \\
\hline Proteasome & 14 & $2.04 \mathrm{e}-12$ \\
\hline Aminoacyl-tRNA biosynthesis & 13 & $3.6 e-11$ \\
\hline $\begin{array}{l}\text { Protein processing in } \\
\text { endoplasmic reticulum }\end{array}$ & 20 & $9.91 e-10$ \\
\hline RNA transport & 18 & $1.52 e-08$ \\
\hline Epstein-Barr virus infection & 17 & $2.89 e-06$ \\
\hline
\end{tabular}

reduction in proteasome activity. However, experiments in cytoplasmic and nuclear extracts showed that neither HS nor NEDD8 knockdown reduced the intrinsic activity of the proteasome (Fig. 3f). In contrast, a small but reproducible increase in nuclear proteasome activity was observed upon NEDD8 knockdown (Fig. 3f). The data suggest that NEDDylation specifically protects the nuclear UPS during the HS response through regulation of protein aggregation.

Proteotoxic stress promotes NEDDylation of RPs. To better understand the role of NEDDylation in UPS function regulation 
Fig. 2 Quantitative proteomics to define the role of NEDD8 in HS-induced protein aggregation. a SILAC proteomics strategy to determine the composition of the HS-induced aggregates. b Scatter plot of the mean of two replicate experiments for the HS SILAC. c Table for the group of proteins with increased aggregation upon HS. The top seven groups are presented, including the number of identified proteins with H/L SILAC ratio $>1$ and the false discovery rate (FDR). d U2OS cells were transfected with control or NEDD8 siRNA or treated with MLN4924 (200 nM) for 48 h, were heat-shocked or not as indicated. Cell extracts were used for western blotting for the indicated proteins. e SILAC strategy to determine the effect of either NEDD8 knockdown or MLN4924 on the composition of HS-induced aggregates. $\mathbf{f}$ Scatter plot comparing the siNEDD8 and MLN4924 SILAC experiments performed in e. Data are the mean of two replicate experiments for each condition. In red and blue are proteins that are either less or more aggregated respectively during HS, specifically upon NEDD8 knockdown but not upon MLN4924 treatment. $\mathbf{g}$ Table for the group of proteins with decreased aggregation upon HS specifically observed upon NEDD8 knockdown

upon proteotoxic stress, our studies focussed on the characterisation of model substrates that aggregate upon HS in an NEDD8-dependent but NAE-independent manner. The proteomic analysis identified RPs as a major group of proteins that fulfil the above criterion (Fig. 2f, g). In addition, RPs have been reported as NEDD8 substrates and more recently implicated in the proteotoxic stress response ${ }^{41,42}$. We initially determined whether RPs found in aggregates upon proteotoxic stress are substrates of NEDDylation. HCT116 cells stably expressing $\mathrm{His}_{6}{ }^{-}$ NEDD8 were heat-shocked or treated with MG132, then subjected to a subcellular fractionation. His $_{6}$-tagged NEDDylated proteins isolated from solubilised aggregates were blotted against RPL7, an RP identified in the proteomics analysis and used as model substrate. The data show that proteotoxic stress increases NEDDylation of RPL7, which is found in the insoluble pellet (Fig. 4a). The use of the specific inhibitors of NAE (MLN4924) and UBA1 (MLN7243) shows that stress-induced NEDDylation of RPL7 depends on UBA1 and not on NAE (Fig. 4b). The UBA1dependent NEDDylation is characterised by the simultaneous modification of the substrate both with NEDD8 and ubiquitin, including the formation of hybrid NEDD8/ubiquitin chains ${ }^{32}$. To determine the modification of RPL7 both with NEDD8 and ubiquitin, we followed a double purification approach. Based on previous studies, we used the UBA domain of DSK2 protein, which in addition to polyubiquitin it also interacts with hybrid NEDD8/ubiquitin conjugates ${ }^{32}$. Extracts of $\mathrm{His}_{6}$-NEDD8-U2OS cells treated with MG132 were used for a GST-UBA pull-down, before eluates were applied in a sequential His ${ }_{6}$-NEDD8 purification under denaturing conditions. Western blot analysis shows the isolation of high molecular weight conjugates of RPL7, which indicates the simultaneous modification of RPL7 with NEDD8/ubiquitin possibly by hybrid chains (Supplementary Figure 6). The detection of mainly high molecular weight RPL7 conjugates is most likely due to the preferential isolation and enrichment of poly-modified conjugates by the UBA domains.

Furthermore, immunofluorescence analysis shows that severe proteotoxic stress (MG132-ON) causes the accumulation of RPL7 within the previously characterised nuclear structures stained with NEDD8 and ubiquitin (Fig. 5a, b). Similar results were obtained for RPL11, also identified in the proteomic analysis (Supplementary Figure 7). As RPs are key components of the nucleolus, we performed costaining of NEDD8 and ubiquitin with other nucleolar proteins, fibrillarin and nucleolin. We found no colocalisation, suggesting that the observed NEDD8/ubiquitin nuclear structures are distinct of the nucleolus, rather a stressinduced nucleolar-related structure composed of RPs (Fig. 5c, d).

To further confirm the role of stress-induced NEDDylation in RPs aggregation we applied an overexpression approach, as previous studies showed that ectopically overexpressed NEDD8 is activated by the ubiquitin UBA1 E1 enzyme. These conditions mirror the stress-induced NEDDylation observed under endogenous levels of NEDD8 expression upon proteotoxic stress ${ }^{32,43}$. Immunofluorescence analysis showed that overexpressed NEDD8 is found in nuclear inclusions in unstressed conditions, similar to the ones observed with endogenous NEDD8 upon HS (Fig. 6a). Importantly, under these conditions NEDD8 overexpression promotes the aggregation of RPL7, although to a smaller extent compared to heat shock (Fig. 6b). In contrast, nucleolin, which was shown in the proteomic analysis (Supplementary Data 1) to aggregate upon HS but independently of NEDD8, was not affected by NEDD8 overexpression (Fig. 6b). In addition, ubiquitin overexpression does not lead to substrate aggregation in the absence of stress (Supplementary Figure 8). Overexpression of the NUB1 (NEDD8 Ultimate Buster 1) protein and its close homologue NUB1L was shown to dramatically repress stressinduced NEDDylation in several cell lines ${ }^{44,45}$. We used NUB1 overexpression as an additional tool to determine the role of stress-induced NEDDylation in protein aggregation. Overexpression of NUB1 reduced both HS-induced NEDDylation and RPL7 aggregation (Fig. 6c). The combination of the above data is consistent with the proteomic analysis, indicating a direct role of stress-induced NEDDylation in promoting nuclear aggregation of RPs upon proteotoxic stress.

HUWE1 is specifically required for stress-induced NEDDylation. While it is established that upon proteotoxic stress NEDD8 is activated by the ubiquitin E1 enzyme leading to hybrid NEDD8/ubiquitin conjugates, the identity of conjugating enzymes that promote NEDDylation during proteotoxic stress remains unknown. Recent studies identified Tom1/HUWE1 as an E3-ligase that is required for RPs ubiquitination upon proteotoxic stress $^{46}$. Our proteomic studies identified HUWE1 as one of the proteins enriched in HS-induced aggregates (Fig. 7a). These data were confirmed by immunofluorescence, which showed that proteotoxic stress causes the appearance of HUWE1 in nuclear aggregates, reminiscent to those observed for NEDD8/ubiquitin (Fig. 7b). We therefore tested the role of HUWE1 in stressinduced NEDDylation. Knockdown of HUWE1 significantly reduced the NEDD8 response to proteotoxic stress, including RPL7 NEDDylation, with no significant effect either on cullin NEDDylation (Supplementary Figure 9) or on the global ubiquitin response to proteotoxic stress (Fig. 7c, d). Consistent with the above, immunofluorescence analysis showed that HUWE1 knockdown caused a dramatic decrease in NEDD8 but not ubiquitin-stained nuclear aggregates upon proteotoxic stress (Fig. 7e, f). The presence of multiple E3-ligases could potentially compensate for the loss of HUWE1, consistent with the small effect of HUWE1 knockdown on global ubiquitination. Thus, the dramatic decrease in stress-induced NEDDylation, including RPL7 as a substrate, observed upon HUWE1 knockdown, suggests that HUWE1 is the major E3-ligase that promotes NEDDylation specifically during proteotoxic stress (Fig. 7c-f).

NEDDylation compromises proteasomal substrate processing. The experiments in Fig. 3 suggested that inhibition of NEDDylation enhances UPS dysfunction during stress. However, this is not due to a decrease in the intrinsic activity of the proteasome 
a

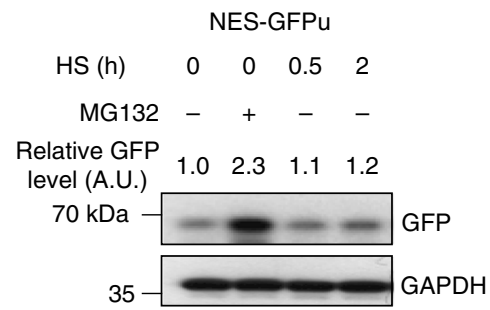

C

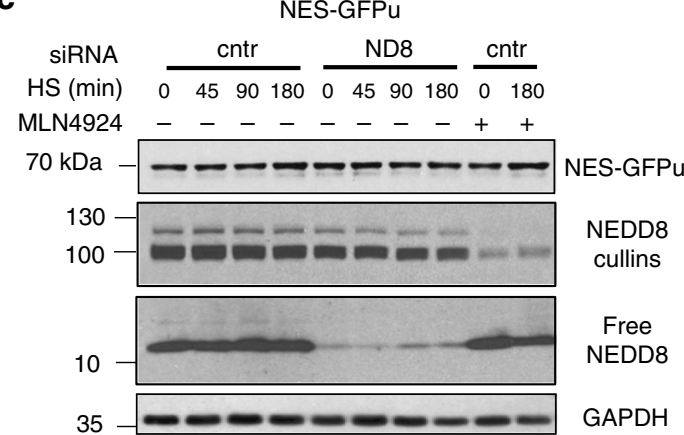

e

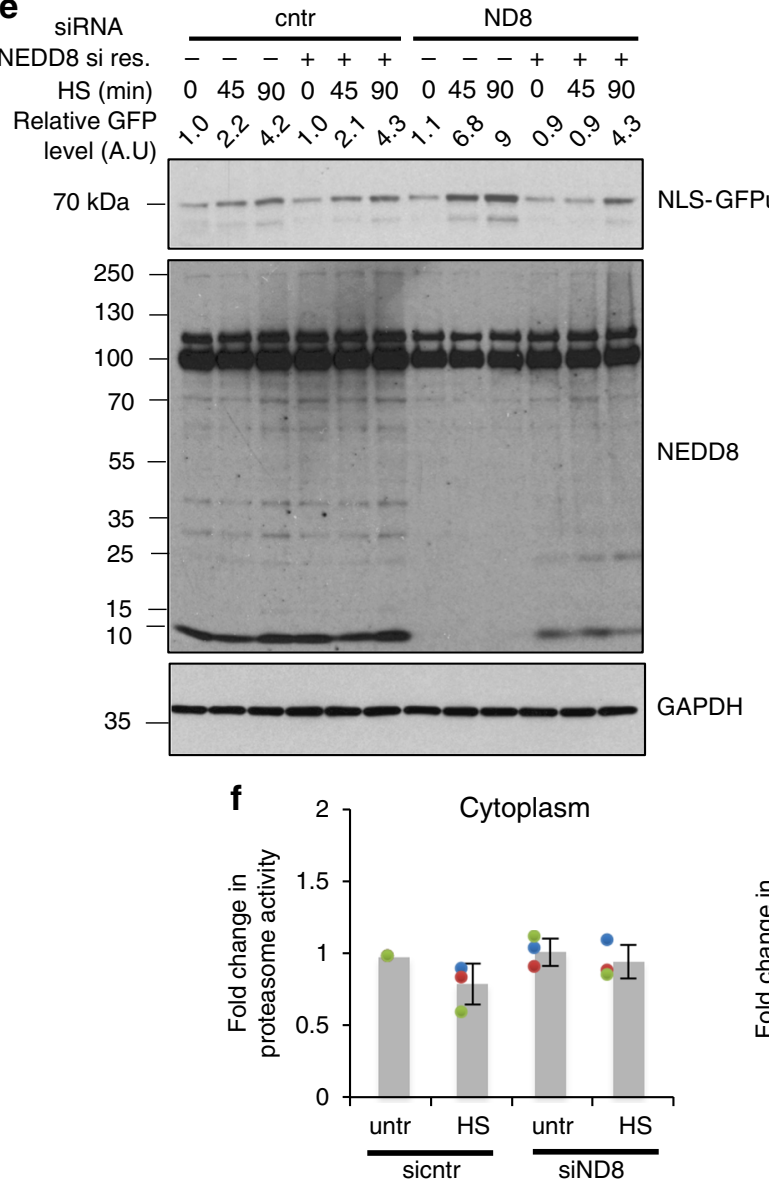

b

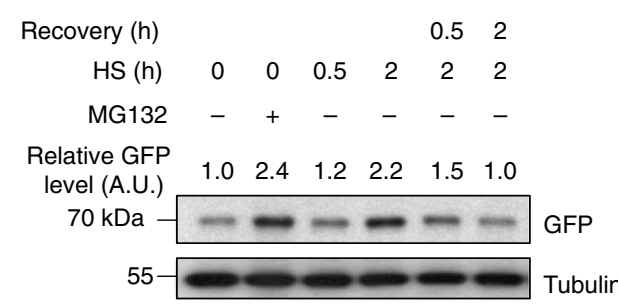

d
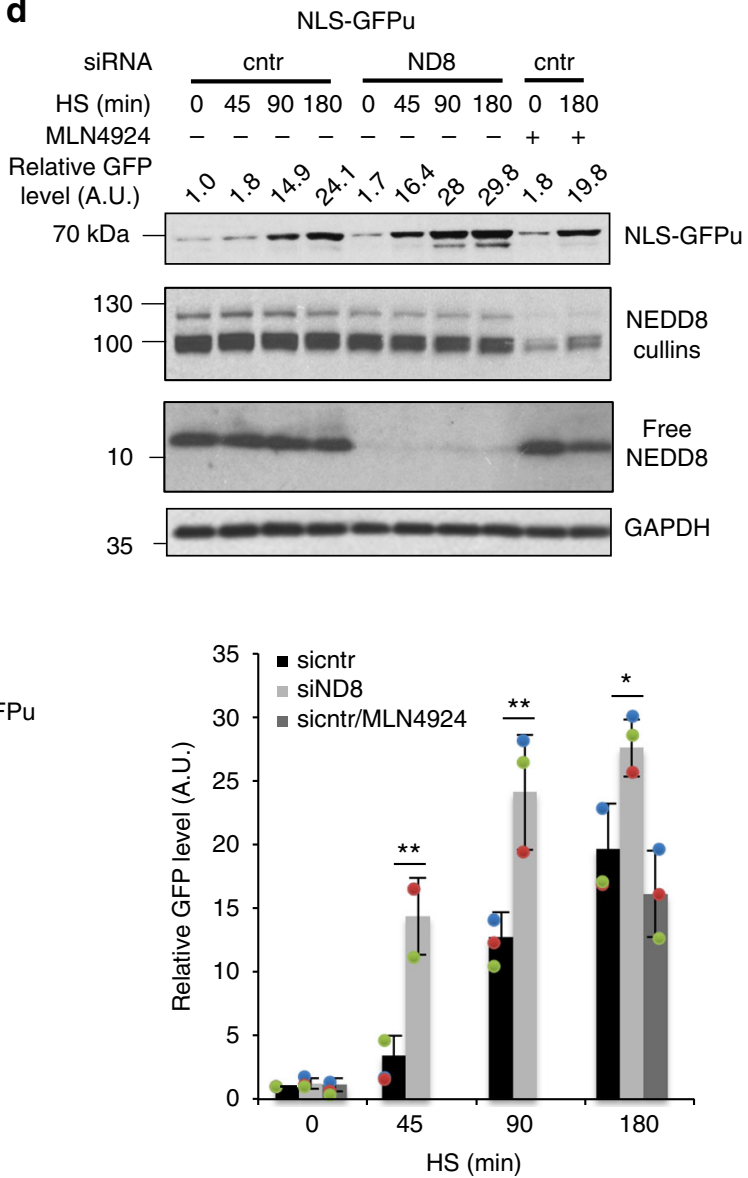

(Fig. 3f), leading us to the hypothesis that NEDDylation could regulate additional steps within the UPS. These include substrate modification and delivery to the proteasome. We used RPL7 as a model substrate to test the above hypothesis. Stress-induced NEDDylation is characterised by the simultaneous modification of substrates with NEDD8 and ubiquitin (Supplementary
Figure 6), raising the possibility that NEDD8 can compromise substrate ubiquitination, the natural targeting signal to the proteasome. Knockdown of NEDD8 increased RPL7 ubiquitination consistent with the idea that NEDD8 conjugation compromises substrate ubiquitination (Fig. 8a). Similar results were obtained upon NUB1 overexpression, which blocks 
Fig. 3 NEDD8 protects the UPS from severe impairment during HS. HEK293 cells stably expressing NES-GFPu (a) or NLS-GFPu (b) were either untreated or heat-shocked at $43^{\circ} \mathrm{C}$. Recovery was performed at $37^{\circ} \mathrm{C}$. MG132 $(25 \mu \mathrm{M}$ for $5 \mathrm{~h})$ was used as positive control for UPS inhibition. Cell extracts were blotted against the indicated proteins. c, d HEK293 cells stably expressing NES-GFPu (c) or NLS-GFPu (d) were transfected with nontarget (cntr) or NEDD8 (ND8) siRNAs, or treated with MLN4924 (200 nM). After $48 \mathrm{~h}$ cells were heat-shocked for the indicated periods and cell extracts were blotted against the indicated proteins. Bottom right graph: Quantitation of three independent experiments performed as in $\mathbf{d} \pm$ SD. Two-tailed unpaired Student's $t$ test, ${ }^{\star} p<0.05,{ }^{\star \star} p<0.01$. e Similar experiment as above using the HEK293 NLS-GFPu cells. Twenty-four hours after siRNA transfection, cells were transfected either with pcDNA3-expressing siRNA-resistant NEDD8 or empty vector. $\mathbf{f}$ U2OS cells transfected with control or NEDD8 siRNA were either untreated or heat-shocked for $30 \mathrm{~min}$. Cells were harvested and proteasome activity in cytoplasmic or nuclear extracts was measured as described in Methods. Graphs represent the average of the fold change in proteasome activity \pm SD from three independent experiments

RPL7-NEDDylation and promotes RPL7-ubiquitination (Supplementary Figure 10A, B). Importantly, the observed increase in RPL7 ubiquitination upon NEDD8 knockdown depends on HUWE1, further confirming the specific role of HUWE1 in RP modification $^{46}$, possibly as a dual E3 using both ubiquitin and NEDD8 for substrate modification (Fig. 8b).

Under similar conditions we monitored the interaction of RPL7 with the proteasome. We used HEK293 cells stably expressing a His-biotinylatable-tagged proteasome subunit (RPN11-HTBH), which is a well-established system to isolate proteasomes ${ }^{47}$. Proteasomes were purified with streptavidin beads as described in Methods and the binding to HA-RPL7 was monitored by western blotting. Exposure of cells to HS induced the RPL7-proteasome interaction, which was further stimulated upon NEDD8 knockdown (Fig. 8c). Repression of NEDDylation by overexpression of NUB1 also promoted RPL7 interaction with the proteasome, especially under HS conditions (Fig. 8d). In these interaction experiments we did not detect modified RPL7, most likely due to rapid deconjugation during the experimentation, as protease inhibitors were not included in order to isolate active proteasomes. The data suggest that proteotoxic stress promotes modification, at least of a fraction, of RPL7 both with NEDD8 and ubiquitin through HUWE1 and prevents targeting of RPL7 to the proteasome. The data do not exclude the possibility that at least for some RPs, targeting to the proteasome is mediated indirectly, through complex formation with other modified RPs. We then followed an alternative approach to determine the potential role of stress-induced NEDDylation in proteasomal degradation. Under conditions of NEDD8 overexpression, which mimic the stress-induced UBA1-dependent NEDDylation of RPL7 (Supplementary Figure 10A), we found that NEDDylated RPL7 has increased stability compared to ubiquitinated RPL7 (Fig. 9a), consistent with the notion that UBA1-dependent NEDDylation compromises substrate degradation. In addition, we isolated from transfected cells either ubiquitin or hybrid NEDD8-ubiquitin conjugates. These conjugates were then used in vitro to monitor their processing/degradation by purified $26 \mathrm{~S}$ proteasomes. While ubiquitin conjugates were efficiently processed/degraded, NEDD8/ubiquitin conjugates were almost completely resistant (Fig. 9b). The combination of the above data strongly indicates that NEDD8 and potentially the formation of hybrid NEDD8/ubiquitin chains compromise substrate processing/degradation by the proteasome.

\section{Discussion}

Defining pathways and mechanisms for the formation and elimination of protein aggregates is an important aspect for our understanding of the response to proteotoxic stress. Here, we identify NEDD8 as regulator of the stress-induced nuclear protein aggregation and UPS function. The data suggest that the NEDD8mediated aggregation protects the nuclear UPS from severe impairment during stress. The finding that both HS and NEDD8 specifically control nuclear but not cytoplasmic UPS activity further supports the notion that NEDD8 is part of the cellular response to control the function of the nuclear UPS during HS.

The nuclear translocation of damaged substrates for proteasomal degradation has been well characterised in yeast and justified by the high efficiency of the nuclear UPS in these model systems $^{48-50}$. However, it is possible that the majority of NEDDylated substrates upon stress are newly synthesised damaged proteins destined for the nucleus. For example, it is known that RPs upon their synthesis are rapidly imported into the nucleus and the nucleolus ${ }^{51}$. High rates of nuclear import for some substrates may compromise recognition, modification and elimination of such damaged proteins in the cytoplasm.

Another critical aspect is the nature of the observed nuclear aggregates. Seminal studies by Latonen et al. identified similar to the presented nuclear structures upon prolonged inhibition of the proteasome activity. They were reported to contain components of the ubiquitin system, cell cycle regulator proteins and polyadenylated RNA but not RNA $^{52}$. More recent studies in Saccharomyces cerevisiae reported stress-induced peri-nucleolar aggregates, which were previously defined as JUNQ ${ }^{53}$. Our proteomics and immunofluorescence analysis indicate that the observed nuclear structures are nucleolus-related (based on protein composition) but not nucleoli, as we failed to detect key nucleolar markers such as fibrillarin and nucleolin. We speculate that these structures represent a late response to protein damage, involving the reorganisation of the nucleolus.

Our current knowledge on protein NEDDylation defines two modes of NEDD8 activation: The so-called canonical via NAE and the atypical via UBA1 observed under proteotoxic stress conditions ${ }^{24,25,44}$. The use of NAE- and UBA1-specific inhibitors and NUB1 overexpression indicates that the identified role of NEDD8 in stress-induced protein aggregation is independent of the canonical NEDD8 pathway. Rather, it has the main characteristics of the atypical NEDDylation, including the simultaneous modification of targets both with NEDD8 and ubiquitin, possibly by hybrid NEDD8/ubiquitin chains. Based on the findings on RPL7, stress-induced NEDDylation could compete with ubiquitin for substrate modification. This can be either at the level of substrate lysine modification site and/or at the level of ubiquitin chains formation. Indeed, proteomic analysis showed that activation of NEDD8 by UBA1 promotes the NEDDylation of ubiquitin at multiple lysine residues including $\mathrm{K} 48^{32}$. In this scenario, activation and conjugation of NEDD8 by enzymes of the ubiquitin system could alter the length and/or topology of ubiquitin chains ${ }^{54}$, or the so-called ubiquitin code. Stress-induced NEDDylation can thus provide a regulatory mechanism that compromises substrate ubiquitination and targeting for proteasomal degradation (Fig. 10). This is supported by the observation that NEDD8 overexpression, which forces NEDD8 conjugation by the ubiquitin system, reduces the rate of substrate degradation (Fig. 9a) and is sufficient to promote substrate aggregation (Fig. 6a, b). In combination with the in vitro analysis showing a clear preference of the $26 \mathrm{~S}$ preoteasome for processing/degrading ubiquitin but not hybrid NEDD8/ubiquitin conjugates, the data 
a
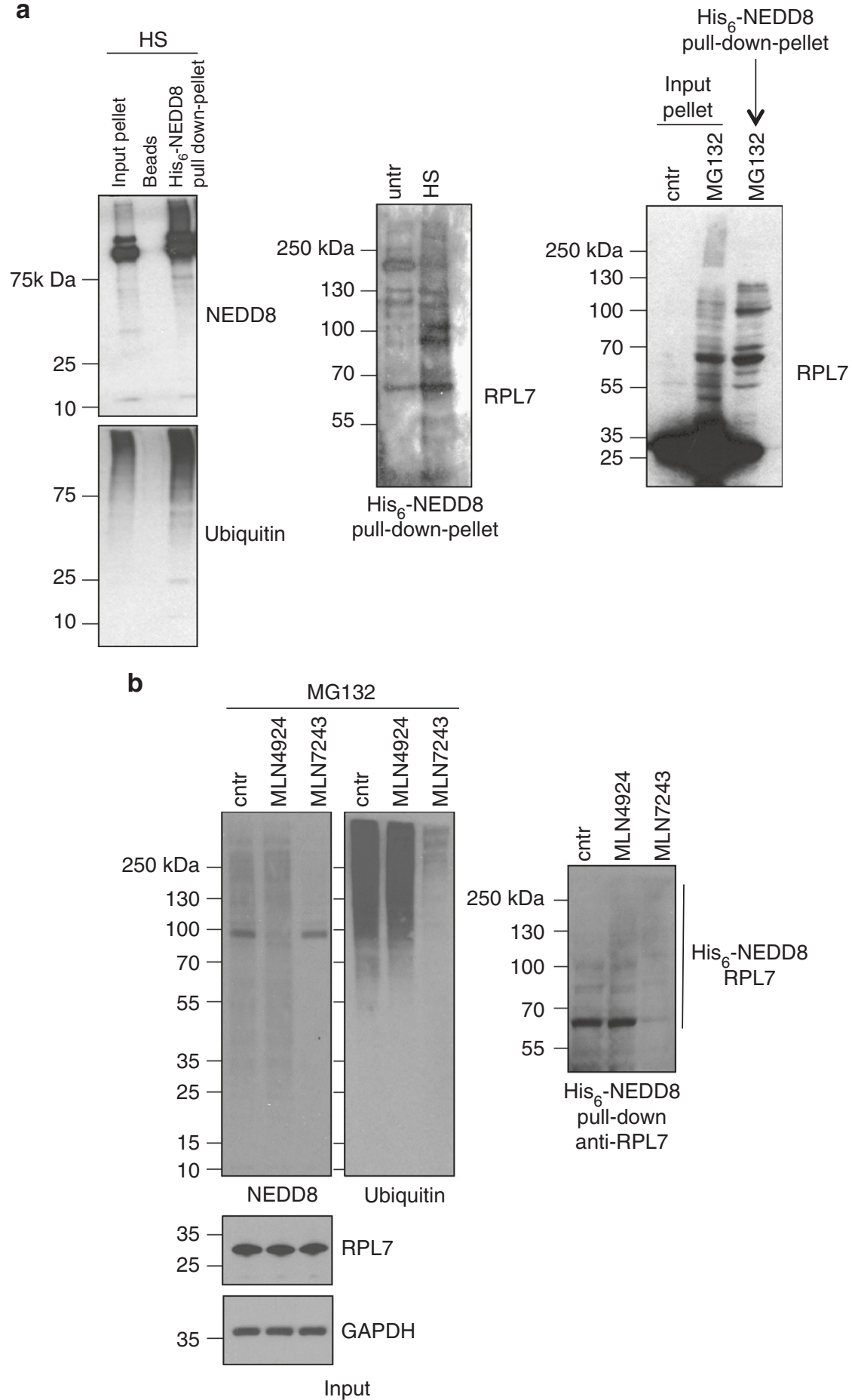

Fig. 4 UBA1-dependent NEDDylation of RPL7 upon proteotoxic stress. a HCT116 cells stably expressing His 6 -NEDD8 were heat-shocked or treated with MG132 $(25 \mu \mathrm{M}, 2 \mathrm{~h})$ and then subjected to a subcellular fractionation. Pellet fractions were solubilized and then NEDDylated substrates were isolated by Ni-NTA pull-down as described in Methods. The isolated NEDDylated substrates were blotted against the indicated proteins. $\mathbf{b}$ Experiment was performed as above but cells were pretreated with MLN4924 (500 nM 15 h) and MLN7243 (500 nM, $3 \mathrm{~h}$ ) before MG132 treatment. Ni-NTA pull-down was performed from total cell extracts and western blot analysis as indicated

indicate that the mechanism by which NEDD8 can promote aggregation of misfolded proteins is to prevent their degradation by the proteasome pathway.

The identification of HUWE1 as the key E3-ligase responsible for stress-induced NEDDylation defines a factor of the ubiquitin system that promotes modification of substrates both with NEDD8 and ubiquitin. These findings in combination with the reported specificity of HUWE1 for RPs upon proteotoxic stress ${ }^{42}$ indicate that RPs may be a key target for stress-induced NEDDylation.

While UBA1-dependent NEDDylation is prominent under proteotoxic stress conditions, we cannot exclude that it also operates under homoeostatic conditions, to a decreased extent. It is however evident that UBA1-dependent NEDDylation is 
a
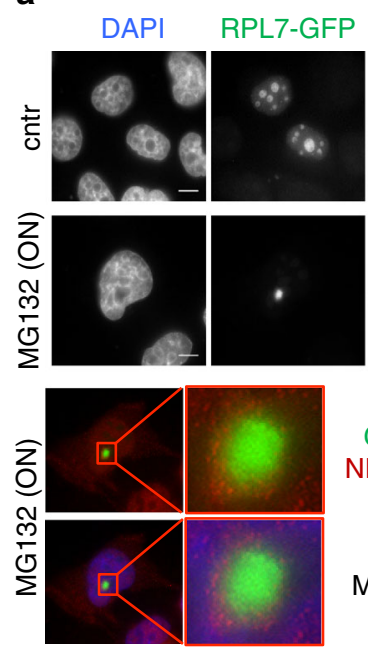

GFP
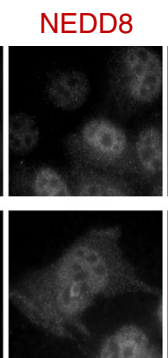

GFP

NEDD8

Merge

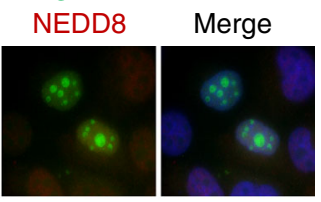

b
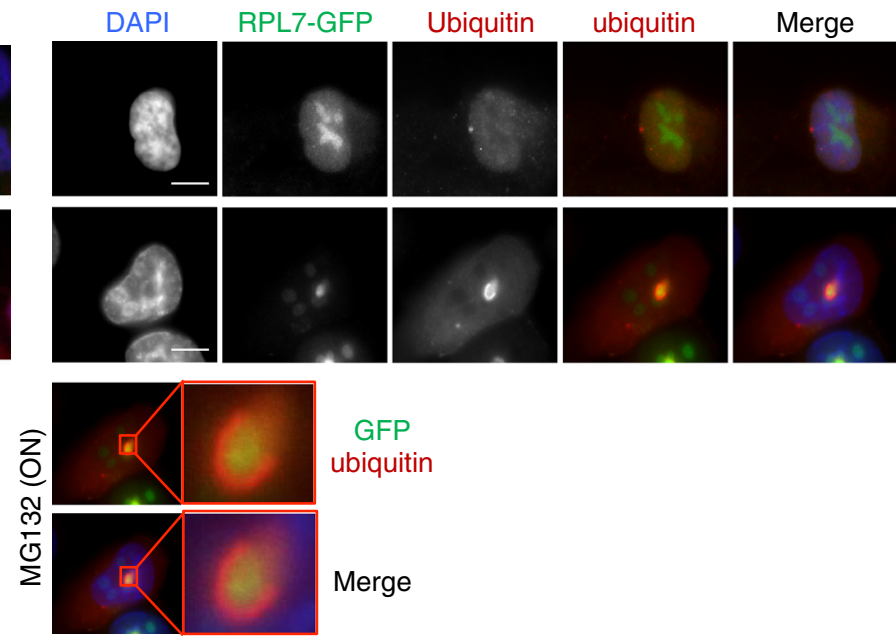

C
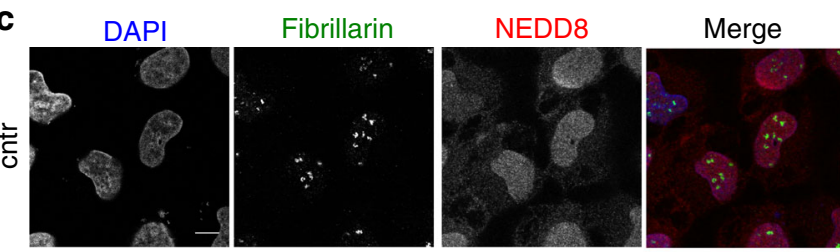

d
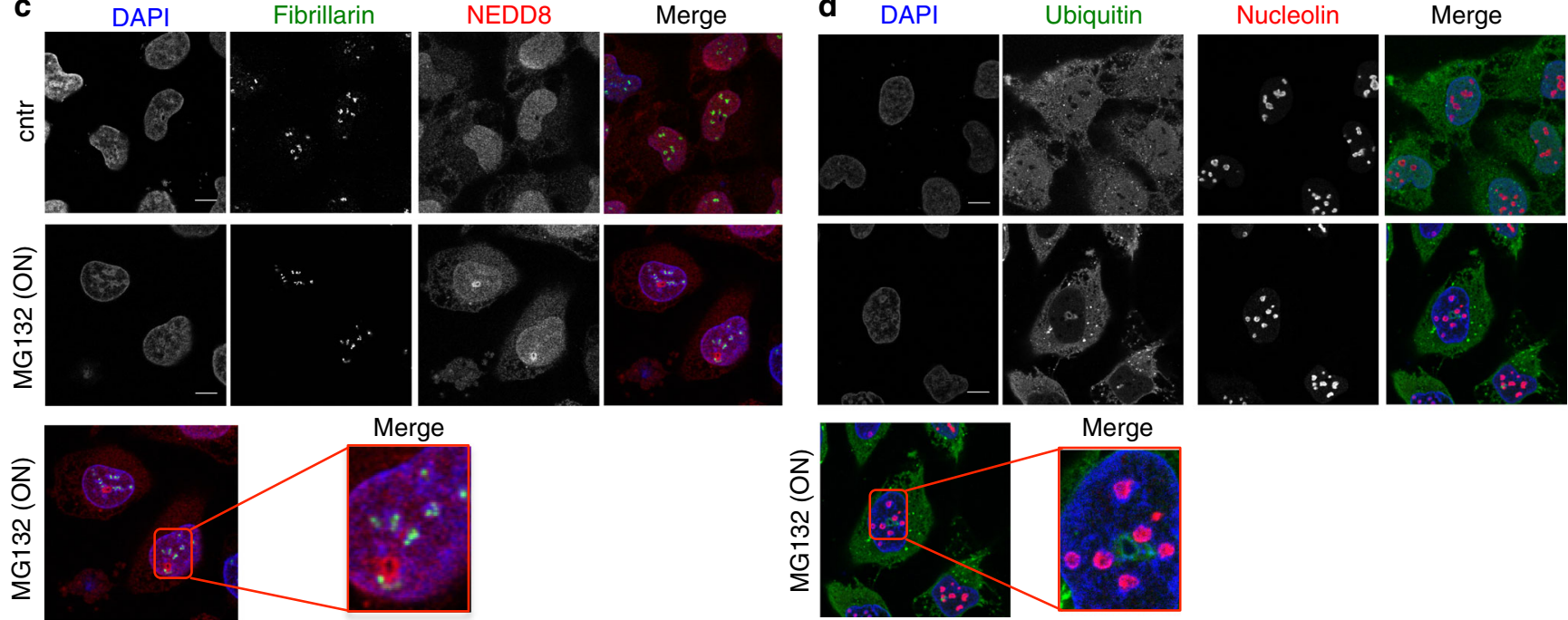

Fig. 5 RPL7 localises within the NEDD8/ubiquitin-stained aggregates. a, b H1299 cells were transfected with RPL7-GFP and 48 h later were treated or not with MG132 ( $5 \mu \mathrm{M}-\mathrm{ON}$ ) and stained for either NEDD8 (a) or ubiquitin (b) (red). Enlarged insets represent the NEDD8/ubiquitin-stained structures observed upon MG132 surrounding RPL7. c, d Experiment performed as above and cells were stained with fibrillarin and NEDD8 (c) or nucleolin and ubiquitin (d). Nuclei were stained with DAPI. Scale bars, $10 \mu \mathrm{m}$

induced and biologically significant when the burden for proteasomal degradation is dramatically increased during proteotoxic stress. Our studies suggest that the transient nuclear aggregation induced by NEDD8 could indeed act as safeguard mechanism for the UPS during stress conditions.

Consistent with studies in S. cerevisiae, and Caenorhabditis elegans $^{46,55,56}$, our proteomic analysis identifies RPs as a major group of proteins enriched in aggregates upon proteotoxic stress. In addition, we found NEDDylation as a regulatory pathway that promotes their aggregation. A critical aspect of RPs biology is that in their free, non-ribosome state, they are susceptible for rapid proteasomal degradation. RPs are constantly being produced in excess over rRNA and are rapidly degraded by the ubiquitin proteasome pathway in the nucleus ${ }^{42,46,51}$. This provides a significant and constant load for nuclear proteasomes, which however can cope with under homoeostatic conditions. Upon proteotoxic stress where the load of misfolded ubiquitinated proteins targeted for proteasomal degradation is dramatically increased, RPs degradation may now become a limiting factor for proper UPS function. Redirection of a fraction of unassembled/ damaged RPs to aggregates via induction of NEDDylation could provide a means to protect nuclear UPS function during stress (Fig. 10). As discussed above, HUWE1 may be the E3-ligase that provides specificity in this response. It also raises the intriguing possibility that an extraribosomal function of RPs is to impact on UPS function and proteostasis. The study is consistent with the model that the main source of toxicity is the soluble unattended misfolded and possibly oligomeric aggregates and that sequestration into transient insoluble deposits is cytoprotective $^{20,21,57,58}$.

\section{Methods}

Cell culture. With the exception of H1299 lung carcinoma cells cultured in RPMI medium the rest of cell lines were cultured in Dulbecco's modified Eagle's medium supplemented with $10 \%$ fetal bovine serum and standard antibiotics (Penicillin, $50 \mathrm{U} / \mathrm{ml}$ and Streptomycin $50 \mu \mathrm{g} / \mathrm{ml}$ ) in $5 \% \mathrm{CO}_{2}$ at $37^{\circ} \mathrm{C}$ in a humidified incubator. HEK293 cells stably expressing NES/NLS-GFPu were a gift from Prof. Ron Kopito and HEK293 RPN11 cells from Dr. Lan Huang. HCT116 and U2OS cells stably expressing wild-type $\mathrm{His}_{6}$-NEDD8 were cultured in the presence of $2.5 \mu \mathrm{M}$ puromycin for selection. Unless otherwise stated, the American Type Culture Collection was the original source of used cell lines. Cell lines have not been authenticated but were routinely tested for mycoplasma contamination and kept in culture for a maximum of 20 passages. 


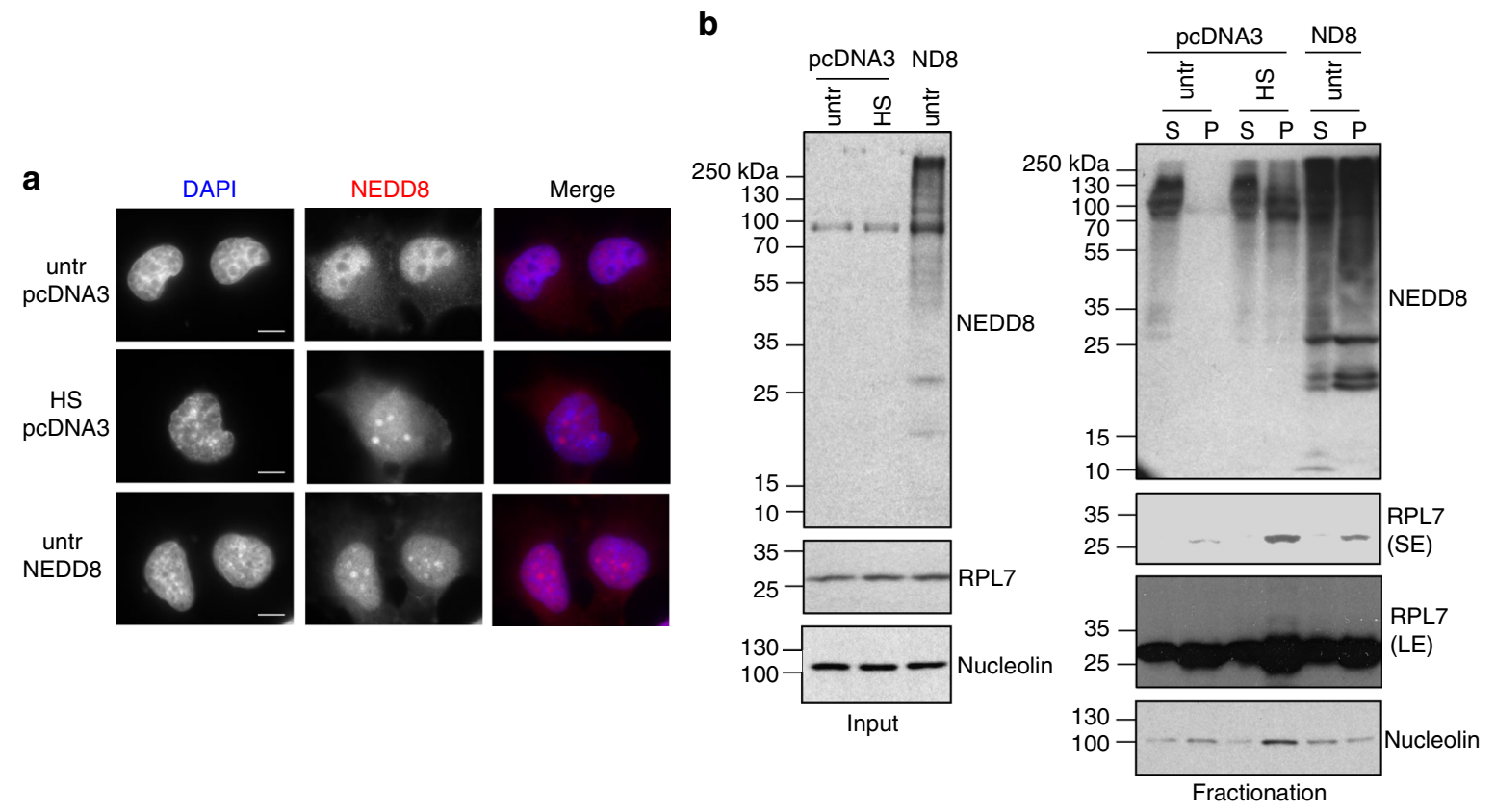

C

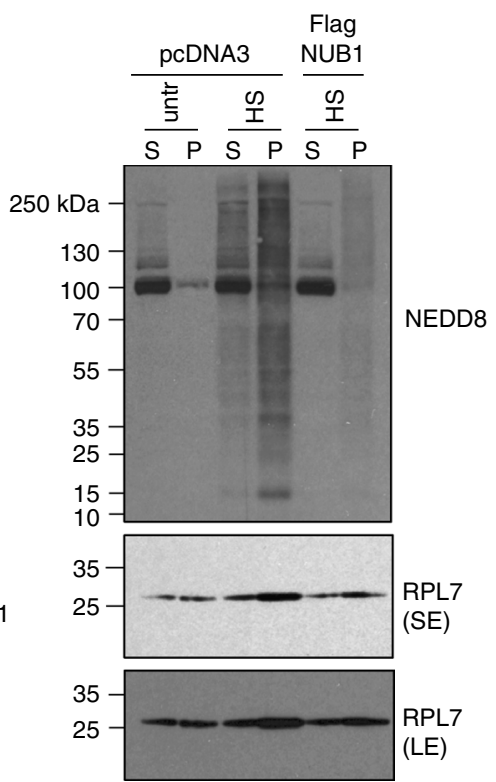

Fig. 6 Overexpression of NEDD8 promotes protein aggregation. a H1299 cells were transfected either with empty pcDNA3 vector or NEDD8 expression constructs. Cells were heat-shocked for $2 \mathrm{~h}$ at $43^{\circ} \mathrm{C}$ and were stained for NEDD8 and DAPI. Scale bars, $10 \mu \mathrm{m}$. b Similar experiment as in a and fractionation was performed to isolate soluble $(S)$ and insoluble proteins from the pellet $(P)$. Western blotting was performed for the indicated proteins

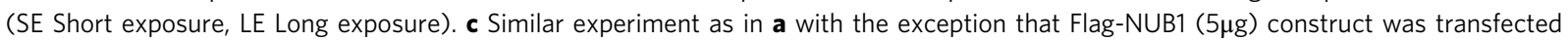

Reagents. Most common chemicals were purchased from Sigma Aldrich. MLN4924 (Takeda Pharmaceuticals), MLN7243 (Chemietek), MG132 (Viva Bioscience), Lipofectamine RNAiMAX (Invitrogen), siRNA On-TARGETplus SMARTpools (Dharmacon), protease Inhibitor Cocktail Tablets EDTA-free, Fugene6 HD (Roche), Suc-LLVY-AMC peptide (BostonBiochem). Rabbit monoclonal anti-NEDD8 (1:2000), Y297 (GeneTex, GTX61205), FK2 mouse anti-ubiquitin, stainings (1:250) (Viva Bioscience, VB2500), rabbit anti-ubiquitin (1:2000), western blotting (DAKO, Z0458), mouse anti-fibrilarin (1:1000) (ab4566), rabbit anti-nucleolin (1:1000) (ab22758), mouse anti-GAPDH (1:5000) (6C5, ab8245), rabbit anti-RPL7 (1:2000) (ab72550) (Abcam), mouse anti-tubulin (1:2000) (Cell Signalling, 3873), mouse anti-HA (1:2000) $(12 \mathrm{C} 5,11583816001)$, mouse anti-GFP (1:500) (11814460001) (Roche), mouse anti-a6 proteasome subunit $(1 \mu \mathrm{g} / \mathrm{ml})$ (Enzo Life Sciences, BML-PW8100), rabbit polyclonal anti-HUWE1 (1:2000) (Bethyl laboratories, A300-486A), mouse monoclonal anti-p21 (1 $\mu \mathrm{g} / \mathrm{ml})(\mathrm{F}-5, \mathrm{sc}-$ 6246, Santa Cruz), rabbit polyclonal anti-CDT1 (1:1000) (\# 06-1295, Millipore), goat anti-mouse Alexa Fluor ${ }^{\circledast} 488$ (115-545-146), goat Anti-Rabbit Alexa Fluor 594 (111-585-008) (Jackson ImmunoResearch).
Transfections. Cells were seeded in six-well plates or $10 \mathrm{~cm}$ dishes to the desired confluency. $5 \mathrm{nM}$ of siRNA was transfected with Lipofectamine RNAiMAX according to the manufacturer's instructions. Nontarget siRNA was used in control transfections. For the SILAC experiment transfections were performed in six-well plates and $6 \mathrm{~h}$ later cells were combined and reseeded in $10 \mathrm{~cm}$ dishes. Cells were harvested $48 \mathrm{~h}$ post-transfection. Fugene6 HD Transfection Reagent was used for plasmid transfections using 3:1 fugene: DNA $(\mu \mathrm{g})$ ratio.

Subcellular fractionation. After the appropriate treatment, cells in $10 \mathrm{~cm}$ culture dish were washed twice with ice-cold PBS, then scrapped into $1 \mathrm{ml}$ PBS. One hundred microliters of cells was pelleted at $16,200 \times g$ for 1 min and lysed with the appropriate volume of $2 \times$ SDS loading buffer. The remaining $900 \mu$ of cells was pelleted at $100 \mathrm{xg}$ for $5 \mathrm{~min}$. Cell pellet was resuspended in $300 \mu \mathrm{l}$ buffer A $(10 \mathrm{mM}$ HEPES-KOH pH 8.0, $10 \mathrm{mM} \mathrm{KCl,} 1.5 \mathrm{mM} \mathrm{MgCl}_{2}$ ) with protease inhibitor and $10 \mathrm{mM}$ iodoacetamide. Cells were lysed by adding Triton-X100 at a final concentration of $0.1 \%$ for $1 \mathrm{~min}$ at $4^{\circ} \mathrm{C}$, then spun for $5 \mathrm{~min}$ at $1300 \times g 4^{\circ} \mathrm{C}$. The 
a

\begin{tabular}{|c|c|c|}
\hline Gene name & $\begin{array}{c}\text { Ratio H/L } \\
\text { HS_Exp. 1 }\end{array}$ & $\begin{array}{c}\text { Ratio H/L } \\
\text { HS_Exp. 2 }\end{array}$ \\
\hline HUWE1 & 3.6596 & 1.6771 \\
\hline
\end{tabular}

C

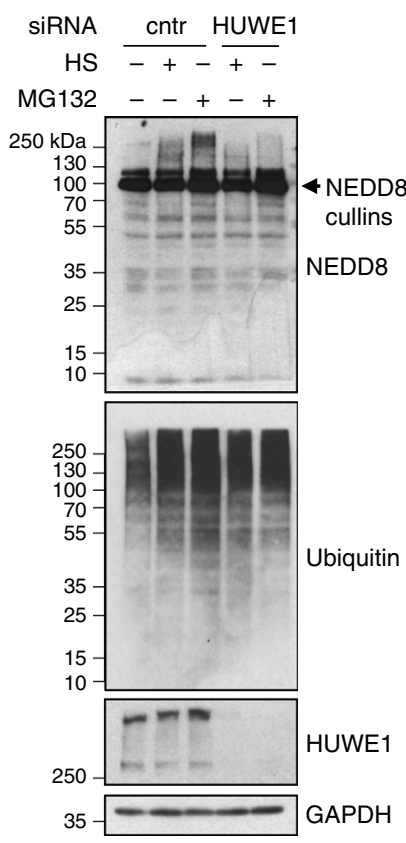

e

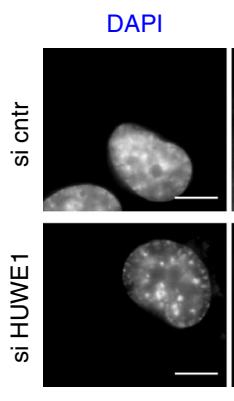

f
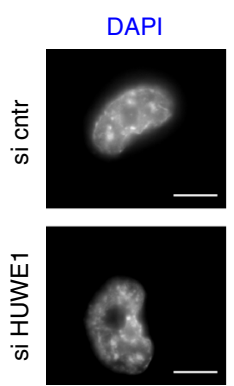

b DAPI
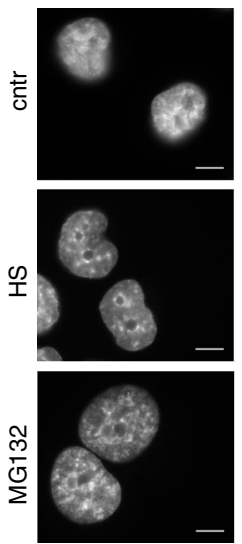

HUWE1
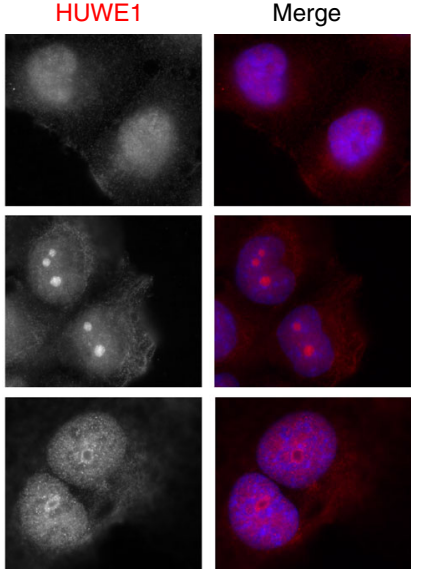

d

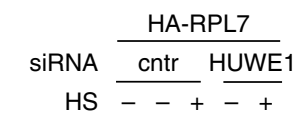

$\mathrm{His}_{6}-\mathrm{NEDD}$

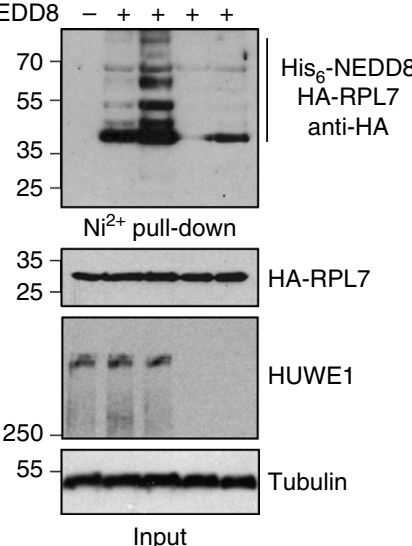

Heat shock

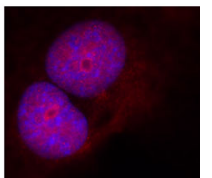

\section{WE1}

HS $\frac{\text { cntr }}{--+} \frac{\text { HUWE }}{-+}$ 
Fig. 7 The HECT E3-ligase HUWE1 is required for stress-induced NEDDylation. a Table showing the SILAC ratio for HUWE1 in the two replicate experiments performed for the aggregate composition upon HS. Heavy, HS; Light, untreated. b H1299 cells were either untreated (DMSO) or exposed to HS $(2 \mathrm{~h})$ or to overnight MG132 $(5 \mu \mathrm{M})$ treatment. Cells were fixed and stained for HUWE1. c U2OS cells were transfected with siRNAs and treated as above. Cell extracts were used for western blotting. $\mathbf{d}$ U2OS cells were transfected with siRNAs before plasmid transfection of HA-RPL7 and His 6 -NEDD8 as indicated. Cells were exposed to HS ( $2 \mathrm{~h}$ ) before Ni-NTA pull-down and western blotting. e, $\mathbf{f} \mathrm{H} 1299$ cells were siRNA transfected as indicated and either heat-shocked (e) or MG132 (f) treated as before. Staining and quantitation of nuclear aggregates stained with NEDD8 or ubiquitin was performed as before. Scale bars, $10 \mu \mathrm{m}$. Values represent the average of three independent experiments \pm SD

Triton X-100, $200 \mathrm{mM}$ iodoacetamide, complete EDTA-free protease inhibitors. After sonication $5 \times 30 \mathrm{~s}$ at $30 \%$ amplitude with $30 \mathrm{~s}$ intervals, insoluble proteins were isolated upon centrifugation at $16,200 \times g, 4{ }^{\circ} \mathrm{C}, 30 \mathrm{~min}$. Pellet was washed $3 \times$ with lysis buffer before being solubilised in $2 \times$ SDS Laemmli's buffer.

\section{Isolation of NEDD8 substrates from the insoluble fraction. For all Ni-NTA} purification experiments from cell extracts we used approximately $0.8 \mathrm{mg}$ of total protein with the exception of the fractionation experiments where $2 \mathrm{mg}$ of total protein was used as starting material. HCT116 cells stably expressing $\mathrm{His}_{6}$-NEDD8 were subjected to a subcellular fractionation after the appropriate treatment as described above. Pellet fractions were washed three times with the buffer $\mathrm{B}$ and then resuspended in $1 \mathrm{ml}$ of $8 \mathrm{M}$ urea, $100 \mathrm{mM} \mathrm{Na} 2 \mathrm{HPO}_{4} / \mathrm{NaH}_{2} \mathrm{PO}_{4}(\mathrm{pH} 8)$, $10 \mathrm{mM}$ Tris- $\mathrm{HCl}(\mathrm{pH} 8)$ and sonicated $4 \times 30 \mathrm{~s}$ at $30 \%$ amplitude (Branson Digital Sonifier). Insoluble particles were eliminated by centrifugation at $18,800 \times g$ for $10 \mathrm{~min}$ at $4^{\circ} \mathrm{C}$. One hundred microliters of each collected supernatant was mixed with equal volume of $2 \times$ SDS loading buffer. The rest was diluted up to $6 \mathrm{ml}$ in the $8 \mathrm{M}$ urea $\mathrm{pH} 8$ buffer with $10 \mathrm{mM}$ Imidazole and $10 \mathrm{mM} \beta$-mercaptoehanol. The purification was then performed using $60 \mu \mathrm{l}$ of Ni-NTA agarose beads according to refs. ${ }^{59,60}$. Briefly binding was performed overnight at $4^{\circ} \mathrm{C}$, before $2 \times$ washes with $8 \mathrm{M}$ urea, pH $8,10 \mathrm{mM} \beta$-mercaptoehanol, $01 \%$ Triton $\mathrm{X}-100$ and $3 \times$ washes with $8 \mathrm{M}$ urea, pH 6.3, $10 \mathrm{mM} \beta$-mercaptoehanol, $01 \%$ Triton X-100. Elution was performed in $2 \times$ SDS Laemmli's buffer containing $250 \mathrm{mM}$ imidazole (room temperature, rotating $20 \mathrm{~min}$ ).

Immunofluorescence microscopy. Cells were seeded on round coverslips $24 \mathrm{~h}$ before treatment. After the HS/MG132 treatment, cells were washed twice with warm PBS and fixed with $4 \%$ formaldehyde for $5 \mathrm{~min}$. Cells were washed three times $(10 \mathrm{~min})$ with warm PBS, and permeabilized with $1 \%$ Triton X-100 in PBS for $10 \mathrm{~min}$. Cells were washed $3 \times 10 \mathrm{~min}$ with PBS before blocking with $0.05 \%$ Tween-20 $+1 \%$ Goat serum in PBS for $1 \mathrm{~h}$. After blockage, cells were incubated with the appropriate primary antibody diluted in $0.05 \%$ tween- $20+1 \%$ Goat serum in PBS for $1 \mathrm{~h}$ at room temperature (or overnight at $4^{\circ} \mathrm{C}$ ). After $3 \times 10 \mathrm{~min}$ washes with $0.05 \%$ Tween-20 in PBS, cells were incubated with corresponding secondary antibodies diluted in $0.05 \%$ Tween- $20+1 \%$ Goat serum in PBS for $1 \mathrm{~h}$ at room temperature in the dark. Samples were washed $3 \times 10 \mathrm{~min}$ with $0.05 \%$ tween-20 in PBS, and then stained with DAPI $(1 / 20,000)$ for $20 \mathrm{~s}$ at room temperature in the dark. Slides were washed $3 \times$ with PBS, mounted with Vectashield Mounting Medium (H-1000, Vector), sealed, and viewed under the microscope Leica DM6000 or Leica SP5-SMD using metamorph software. The images were analysed by ImageJ64 software. The 3D rendering experiment was performed from Z-stacks (Leica SP5-SMD) using Imaris 8.3.1 (Bitplane an Oxford Instruments company). The colocalisation between NEDD8 and ubiquitin in the nuclear structures was analysed as described in refs. ${ }^{61,62}$. Briefly, quantifications of colocalisation and determination of Pearson's correlation coefficient were performed using the ImageJ JaCoP plugin. For each channel the plot profile was acquired and individual measurements were exported as excel file to make the presented graph. All primary antibodies were used at 1:250 dilution with the exception of antiNEDD8 (1:150). The secondary antibodies Alexa goat anti-mouse 488 was diluted at 1:500, the goat anti-rabbit 594 1:500.

Western blot analysis. For all inputs we routinely used approximately $20 \mu \mathrm{g}$ of total protein. Proteins were resolved in $4-12 \%$ precast Bis-Tris gels and transferred onto PVDF membrane using the Bio-Rad Mini Trans-Blot apparatus. Membranes were blocked in $5 \%$ milk solution (PBS with $0.1 \%$ Tween- 20 and $5 \%$ skimmed milk) for $1 \mathrm{~h}$ at room temperature with gentle agitation. Membranes were incubated with the primary antibodies overnight at $4{ }^{\circ} \mathrm{C}$. Primary antibodies were diluted in PBS $0.1 \%$ Tween-20 with $3 \%$ BSA and $0.1 \% \mathrm{NaN}_{3}$. Membranes were washed $3 \times 10 \mathrm{~min}$ with PBS $0.1 \%$ Tween-20 and then incubated with the appropriate secondary antibody (1:2000) (Sigma Aldrich) for $1 \mathrm{~h}$ at room temperature (5\% milk). After incubation, membranes were washed $2 \times 15$ min with PBS $0.1 \%$ Tween- 20 followed by $2 \times 5$ min with PBS. Detection was performed with ECL Western Blotting Detection Reagents and membranes were exposed to X-ray Medical Film before being developed. Quantifications were performed with ImageJ. Fully uncropped scans of blots can be found in Supplementary Figure 11.

SILAC-mass spectrometric analysis. Cells were labelled either with light (Lys0/ Arg0) or heavy (Lys8/Arg10) amino acids. Cells grown in light medium were left at $37^{\circ} \mathrm{C}$ whereas cells in heavy medium were heat-shocked at $43^{\circ} \mathrm{C}$ for $2 \mathrm{~h}$. In the experiment of NEDD8 knockdown and MLN4924 treatment, control and siNEDD8-transfected cells or control siRNA transfected and MLN4924-treated cells were all heat-shocked at $43^{\circ} \mathrm{C}$ for $2 \mathrm{~h}$. For each condition $10 \times 10 \mathrm{~cm}$ dishes of $80 \%$ confluence were collected in PBS counted and equal number of cells were mixed. Upon isolation of insoluble proteins (see subcellular fractionation), $50 \mu \mathrm{g}$ of protein were run for $15 \mathrm{~min}$ on $4-12 \%$ precast NuPAGE and coomassie blue stained. Lanes were cut in two gel pieces and in-gel trypsin digestion was performed as described in ref. ${ }^{63}$. Peptides were analysed online by nano-flow HPLCnanoelectrospray ionisation using a Qexactive Plus mass spectrometer (Thermo Fisher Scientific) coupled to a nano- LC system (U3000-RSLC, Thermo Fisher Scientific). Desalting and preconcentration of samples were performed online on a Pepmap ${ }^{\circledast}$ precolumn $(0.3 \times 10 \mathrm{~mm}$; Dionex $)$. A gradient consisting of $0-40 \% \mathrm{~B}$ in A for $140 \mathrm{~min}$ (A: $0.1 \%$ formic acid, $2 \%$ acetonitrile in water, and B: $0.1 \%$ formic acid in acetonitrile) at $300 \mathrm{nl} / \mathrm{min}$ was used to elute peptides from the capillary reverse-phase column $\left(0.075 \times 150 \mathrm{~mm}\right.$, Pepmap ${ }^{\circledR}$, Dionex $)$. Data were acquired using the Xcalibur software (version 4.0). A cycle of one full-scan mass spectrum $(375-1500 \mathrm{~m} / \mathrm{z})$ at a resolution of 70,000 (at $200 \mathrm{~m} / \mathrm{z}$ ), followed by 12 datadependent MS/MS spectra (at a resolution of 17,500, isolation window $1.2 \mathrm{~m} / \mathrm{z}$ ) was repeated continuously throughout the nanoLC separation. Raw data analysis was performed using the MaxQuant software (version 1.5.5.1) with standard settings. Used database consists of Human entries from Uniprot (reference proteome UniProt 2017_03) and 250 contaminants (MaxQuant contaminant database). Relative proteins quantifications were calculated on the median SILAC ratios. For further analysis we consider only proteins with at least two peptides. Perseus (version 1.5.5.1) was used for graphical representation of the data, based on the mean value of $\log 2$ ratio (only for proteins quantified in duplicate) after elimination of contaminants and reverse entries. Functional enrichment on KEGG pathway was determined using STRING database with a confidence interaction level of 0.7 . The False Discovery Rate was calculated from the STRING database analysis on overrepresented KEGG pathways with a confidence interaction level of 0.7 .

35S-Methionine labelling and immunoprecipitations. Cell labelling was performed as described ${ }^{41}$. Briefly, cells were seeded in $6 \mathrm{~cm}$ plates and serum starved for $1 \mathrm{~h}$ with Methionine/Cysteine-free medium. $350 \mu \mathrm{Ci} /$ plate of ${ }^{35} \mathrm{~S}$-Methionine was added and cells were either incubated at $37^{\circ} \mathrm{C}$ or heat-shocked for $20 \mathrm{~min}$. Cells were washed in PBS, lysed in $400 \mu$ l of NP40 lysis buffer and extracts were precleared with protein G beads or GST beads. Immunoprecipitations were performed with $2 \mu \mathrm{l}$ of NEDD8 antibody or $5 \mu \mathrm{g}$ of GST-GFPtrap (in house) and $20 \mu \mathrm{l}$ of either protein $\mathrm{G}$ beads or GST beads overnight before washing the beads $3 \times$ with $500 \mu \mathrm{l} \mathrm{NP}-40$ lysis buffer. Samples were eluted in $2 \times$ SDS buffer, boiled for $5 \mathrm{~min}$ and analysed by SDS-PAGE. Gels were dried and exposed to X-ray films overnight at $-70^{\circ} \mathrm{C}$.

Sequential GST-UBA/ Ni-NTA purification. $4 \times 15 \mathrm{~cm}$ dishes of U2OS cells stably expressing $\mathrm{His}_{6}$-NEDD8 were treated with $25 \mu \mathrm{M}$ of MG132 for $3 \mathrm{~h}$ before harvesting in PBS. Cells were lysed in $1 \mathrm{ml}$ of $50 \mathrm{mM}$ Tris- $\mathrm{HCl} \mathrm{pH} \mathrm{7.4,150} \mathrm{mM}$ $\mathrm{NaCl}, 1 \% \mathrm{NP} 40,10 \%$ glycerol, $10 \mathrm{mM}$ EDTA, $50 \mathrm{mM}$ iodoacetamide and protease inhibitors (Roche), by syringing 15 times and incubation on ice for $30 \mathrm{~min}$. Upon centrifugation at $16,200 \times g$ for $15 \mathrm{~min}$, supernatant was incubated overnight at $4{ }^{\circ} \mathrm{C}$ with $10 \mu \mathrm{g}$ of GST-UBA (DSK2 protein-in house) and $30 \mu \mathrm{l}$ of prewashed (lysis buffer) GST beads. Beads were washed $3 \times$ with $500 \mu$ lysis buffer (with no protease inhibitors and iodoacetamide) and split in two equal parts. One was used to elute proteins in $2 \times$ SDS loading buffer for the UBA input and the other was used to elute proteins (15 min, room temperature) in $500 \mu$ of $8 \mathrm{M}$ urea, $100 \mathrm{mM}$ $\mathrm{Na}_{2} \mathrm{HPO}_{4} / \mathrm{NaH}_{2} \mathrm{PO}_{4}, 10 \mathrm{mM}$ Tris- $\mathrm{HCl}$ pH 8 (Buffer I). The urea eluates were incubated with $50 \mu \mathrm{l} \mathrm{Ni-NTA}$ agarose beads for $4 \mathrm{~h}$ at room temperature. Beads were washed $5 \times$ with $500 \mu$ of Buffer I and His ${ }_{6}$-NEDD8 conjugates were eluted with $200 \mu \mathrm{l}$ of Buffer I, $250 \mathrm{mM}$ imidazole, $15 \mathrm{~min}$ at room temperature.

Quantitative real-time RT-PCR. Total RNA isolation and SYBR qPCR was performed as described ${ }^{64}$ using the following primers:

GFP-F: 5'-ACGTAAACGGCCACAAGTTC

GFP-R: 5'-AAGTCGTGCTGCTTCATGTG

nedd8-F: 5' - ATGCTAATTAAAGTGAAGAC

nedd8-R: 5'-TCCTCCTCTCAGAGCCAACAC

Briefly, six-well plates were used to isolate total RNA using the SV total RNA isolation kit (Promega). Five hundred nanograms of RNA was used to prepare 
a

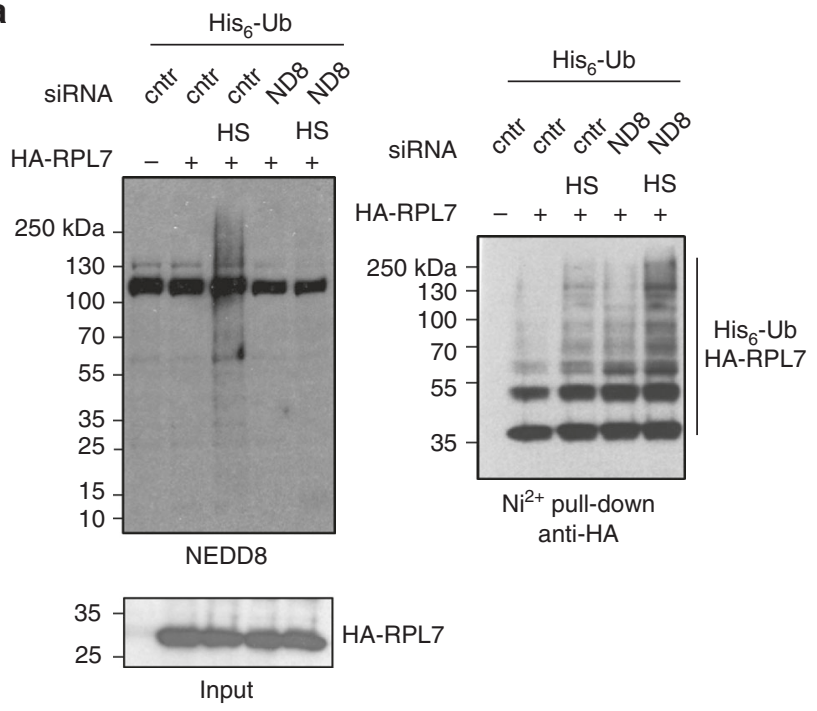

b

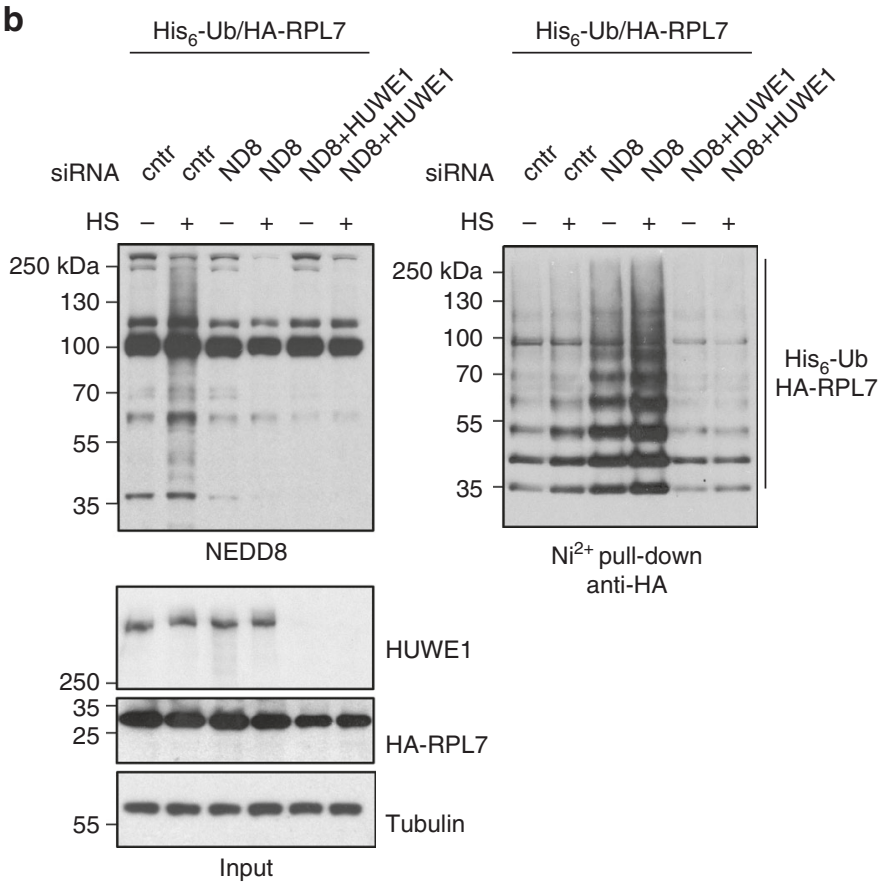

d

C

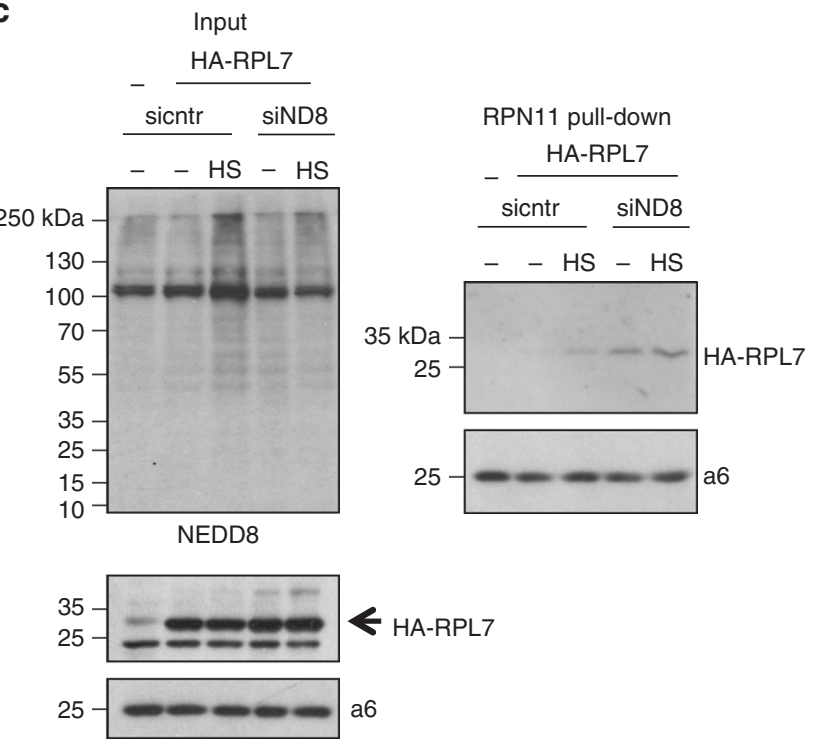

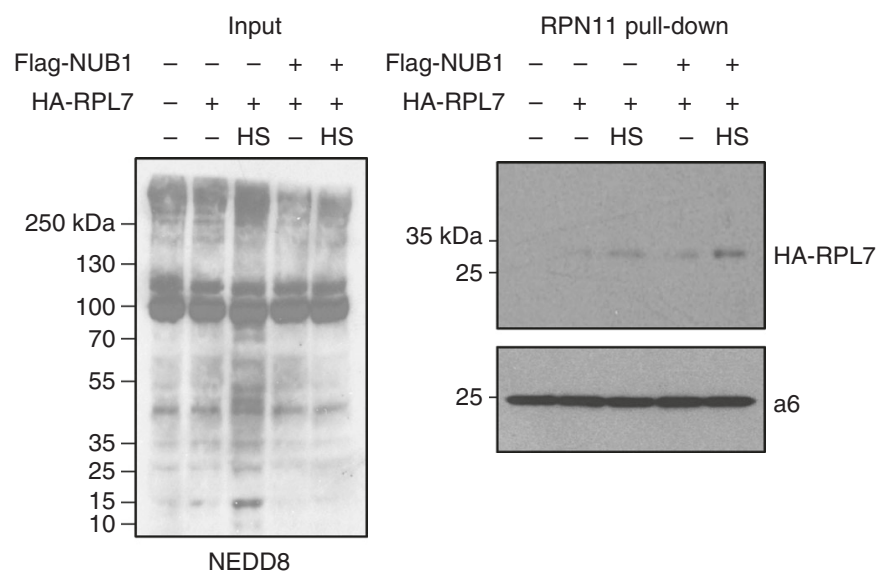

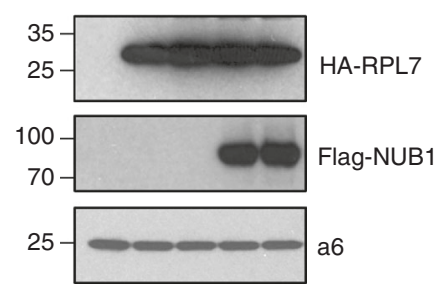

Fig. 8 NEDDylation compromises substrate ubiquitination and targeting to the proteasome. a U2OS cells were first transfected with control or NEDD8 siRNA and $24 \mathrm{~h}$ later with $\mathrm{His}_{6}$-ubiquitin and HA-RPL7 plasmids as indicated. Twenty-four hours later cells were heat-shocked for 45 min and extracts were used for Ni-NTA pull-downs. Western blotting on purified His 6 -ubiquitin conjugates and total cell extracts was performed for the indicated proteins. b Similar experiment as in (a) but cells were cotransfected with the HUWE1 siRNA as indicated. c HEK293 cells stably expressing RPN11-HTBH were transfected with control or NEDD8 siRNA and $24 \mathrm{~h}$ later with RPL7 expressing construct. Twenty-four hours later cells were heat-shocked for 45 min and proteasomes were purified with streptavidin beads. Total cell extracts or biotin pull-downs were blotted against the indicated proteins. $\mathbf{d}$ Similar experiment as in (c) with the exception that Flag-NUB1 or pcDNA3 $(5 \mu \mathrm{g})$ constructs were transfected instead

cDNA with oligo dT using the Transcriptor High Fidelity cDNA Synthesis kit (Roche). $2 \mu \mathrm{l}$ of $10 \times$ diluted cDNA was used in $12 \mu \mathrm{l}$ qPCR reactions with the appropriate primers and SYBR Green PCR Master Mix (Applied Biosystems).

Proteasome activity assay in cell extracts. Proteasome activity in cell extracts was performed according to ref. ${ }^{65}$. U2OS cells in six-well plates were transfected with nontarget or NEDD8 siRNA before reseeded in $10 \mathrm{~cm}$ plates. Cells were either untreated or heat-shocked for $30 \mathrm{~min}$ and cells were harvested in PBS and lysed in hypotonic buffer containing $10 \mathrm{mM}$ HEPES, pH 7.9, $1.5 \mathrm{mM} \mathrm{MgCl}_{2}, 10 \mathrm{mM} \mathrm{KCl}$, $0.5 \mathrm{mM}$ DTT, $1 \mathrm{mM}$ ATP, $0.005 \%$ digitonin for $2 \mathrm{~min}$ on ice. Cells were centrifuged at $1200 \times g$ for $5 \mathrm{~min}$ at $4{ }^{\circ} \mathrm{C}$, the supernatant (cytoplasm) was transferred to fresh tubes and pellet was briefly washed with the lysis buffer, passed through a sucrose cushion before lysed in the hypotonic buffer with $0.05 \%$ digitonin to obtain the nuclear extract $(10 \mathrm{~min}$ on ice). Samples were centrifuged at $16,200 \times \mathrm{g}$ for $10 \mathrm{~min}$ at $4{ }^{\circ} \mathrm{C}$ and supernatant (nuclear extracts) were transferred to fresh tubes. $10-20 \mu \mathrm{g}$ 
a
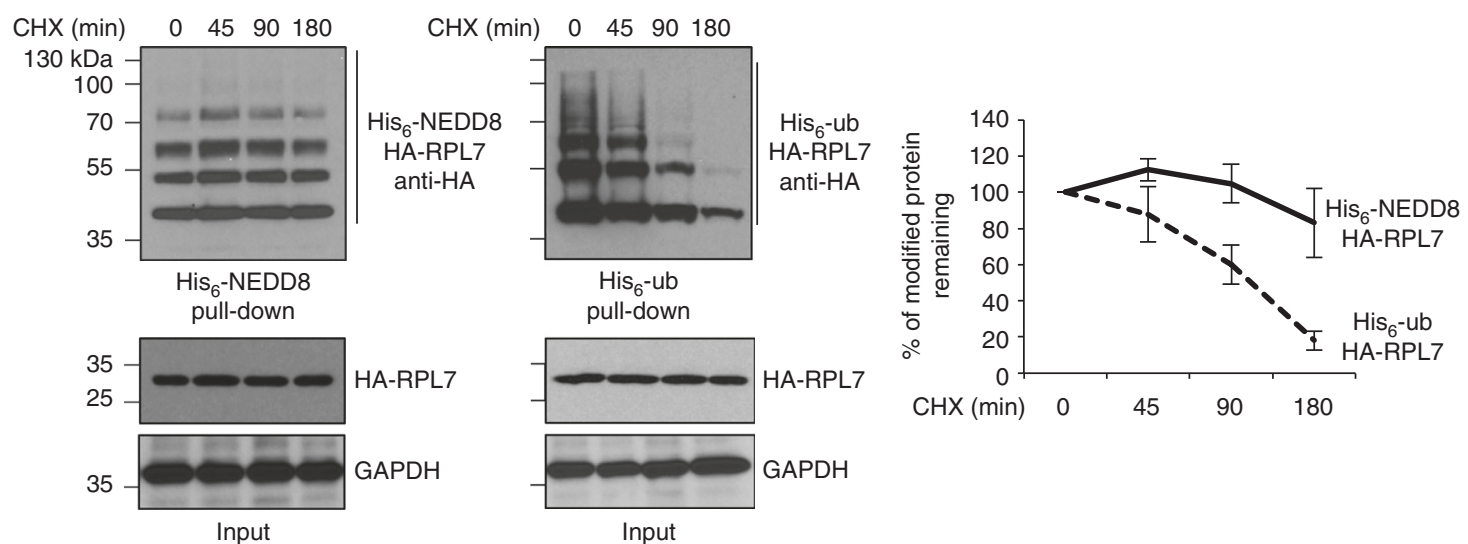

b

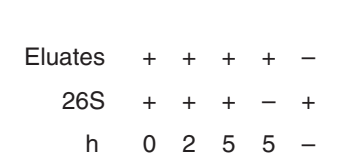

$\mathrm{His}_{6}-\mathrm{Ub}$

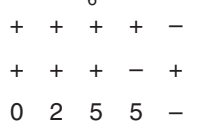

$++++-$

$+++-+$

0255 -
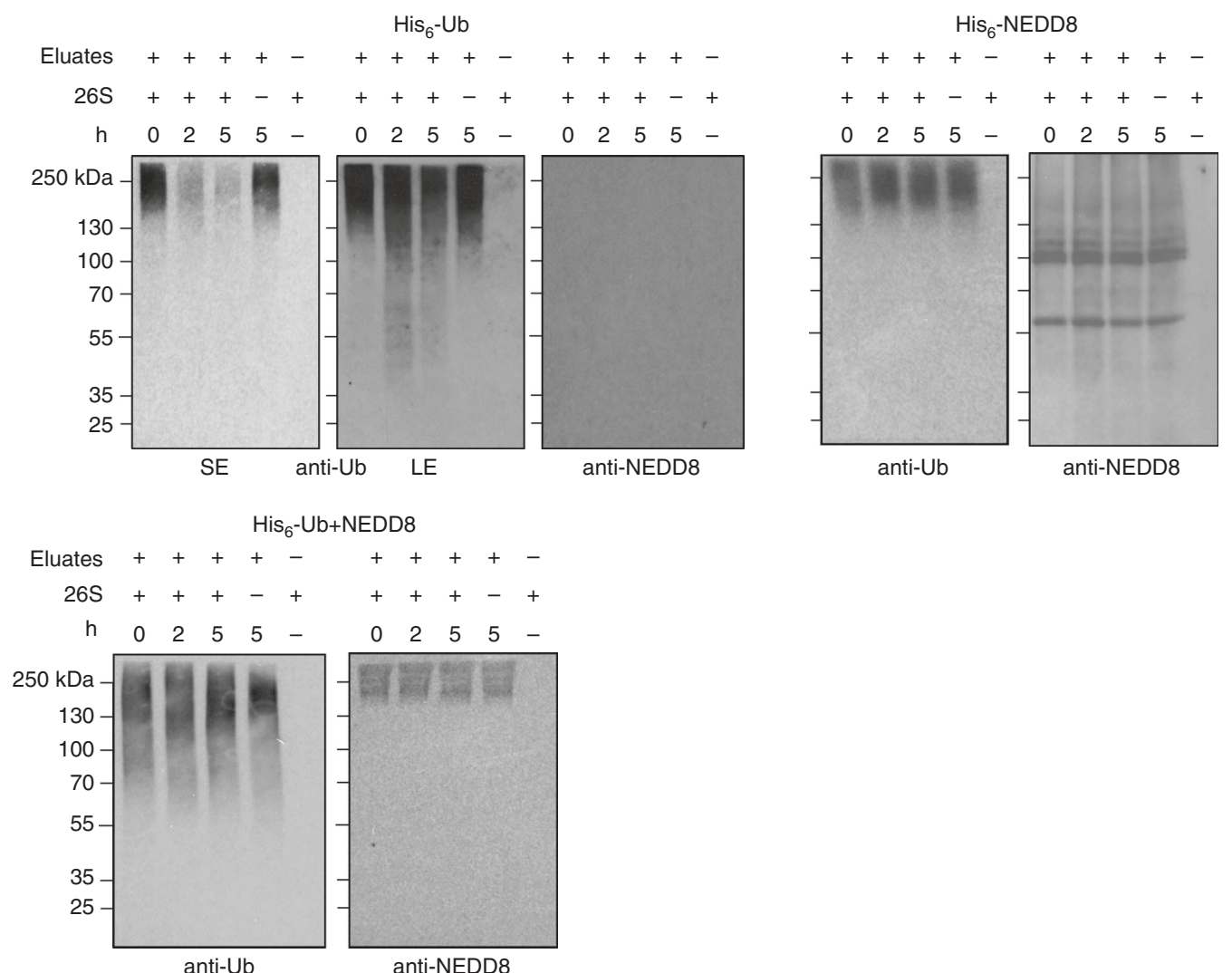

anti-NEDD8

anti-Ub

anti-NEDD8
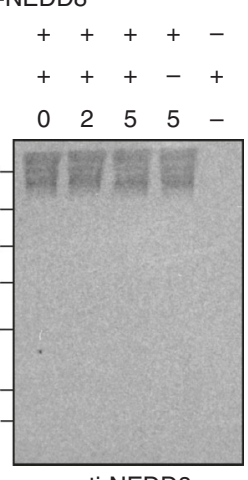

anti-NEDD8

Fig. 9 NEDDylation compromises proteasomal degradation of substrates. a U2OS cells were transfected with $3 \mu \mathrm{g}$ of HA-RPL7 and $4 \mu \mathrm{g}$ of His 6 -NEDD8 or $\mathrm{His}_{6}$-ubiquitin. Forty-eight hours post-transfection cycloheximide $(\mathrm{CHX}, 100 \mu \mathrm{g} / \mathrm{ml})$ was added and cells were harvested at the indicated time points, before lysis and Ni-NTA pull-down. The signal in each lane within the length indicated by the bar was used for quantification. The graph represents the average values of three independent experiments \pm SD. $\mathbf{b} \mathrm{His}_{6}$-ubiquitin or $\mathrm{His}_{6}$-NEDD8 conjugates were isolated as described in Methods and used in vitro in a $26 \mathrm{~S}$ proteasome processing/degradation assay. Western blotting was performed with the indicated antibodies (SE short exposure, LE long exposure)

of total protein was used in a proteasome assay containing $50 \mathrm{mM}$ Tris- $\mathrm{HCl} \mathrm{pH}$ 7.5, $5 \mathrm{mM} \mathrm{MgCl}, 1 \mathrm{mM} \mathrm{ATP,}, 100 \mu \mathrm{M}$ of the substrate (Suc-LLVY-AMC). Assays were performed in 96 -well plates for a period of $30 \mathrm{~min}$. The proteasome inhibitor MG132 was used to measure any nonproteasome protease activity and was subtracted from the data.

Isolation of conjugates for the in vitro proteasome assays. $3 \times 10 \mathrm{~cm}$ dishes of HEK293 cells for each condition were transfected with $\mathrm{His}_{6}{\text {-ubiquitin, } \mathrm{His}_{6}{ }^{-}}^{-}$ NEDD8 or untagged NEDD8 ( $2 \mu \mathrm{g}$ each) with the calcium phosphate method as indicated. Forty-eight hours post transfections cells were harvested and lysed in: $25 \mathrm{mM}$ Tris- $\mathrm{HCl}, \mathrm{pH} 7.5,500 \mathrm{mM} \mathrm{NaCl}, 0.1 \%$ Triton X-100, $1 \times$ protease inhibitors (Roche), $50 \mathrm{mM}$ iodoacetamide, incubated on ice for $15 \mathrm{~min}$. After centrifugation at $16,200 \times g, 4^{\circ} \mathrm{C}$ for $15 \mathrm{~min}$, the supernatant was incubated with $50 \mu \mathrm{l}$ of Ni-NTA agarose beads, prewashed in lysis buffer, overnight at $4{ }^{\circ} \mathrm{C}$. Beads were extensively washed $5 \times$ with $1 \mathrm{ml}$ of $25 \mathrm{mM}$ Tris- $\mathrm{HCl}, \mathrm{pH} 7.5,1 \mathrm{M} \mathrm{NaCl}, 0.1 \%$ Triton X-100 over a period of $5 \mathrm{~h}$ in total at $4{ }^{\circ} \mathrm{C}$, before another $3 \times$ washes with the same wash buffer with no Triton X-100. His $_{6}$-tagged conjugates were eluted twice with $100 \mu \mathrm{l}$ (each) of $25 \mathrm{mM}$ Tris-HCl, pH 7.5, $500 \mathrm{mM} \mathrm{NaCl}, 500 \mathrm{mM}$ imidazole by rotating at $4{ }^{\circ} \mathrm{C}$ for $20 \mathrm{~min}$. Eluates were combined and dialysed overnight at $4^{\circ} \mathrm{C}$ against 21 of: $25 \mathrm{mM}$ Tris- $\mathrm{HCl}, \mathrm{pH} 7.5,10 \mathrm{mM} \mathrm{MgCl}_{2} .1 \mathrm{mM}$ of DTT was added to dialysed samples and directly used in vitro in a $26 \mathrm{~S}$ proteasome assay.

In vitro proteasome processing/degradation assays. $26 \mathrm{~S}$ proteasomes were purified from HEK293 cells stably expressing RPN11-HTBH with streptavidin beads as described in ref. ${ }^{47}$. Briefly, cells from $10 \times 15 \mathrm{~cm}$ plates were harvested and lysed in lysis buffer: $100 \mathrm{mM} \mathrm{NaCl}, 50 \mathrm{mM}$ sodium phosphate $\mathrm{pH} 7.5,10 \%$ glycerol, $5 \mathrm{mM}$ ATP, $1 \mathrm{mM}$ DTT, $5 \mathrm{mM} \mathrm{MgCl}_{2}$, $1 \times$ protease inhibitors (Roche) and $0.5 \% \mathrm{NP}-40$. Streptavidin beads $(100 \mu \mathrm{l})$ prewashed in lysis buffer were added to cell extracts and incubated overnight at $4{ }^{\circ} \mathrm{C}$. Beads were washed with $1 \mathrm{ml}$ of the lysis buffer, followed by two washes with $1 \mathrm{ml}$ of wash buffer $(50 \mathrm{mM}$ Tris- $\mathrm{HCl}, \mathrm{pH}$ 7.5, $10 \%$ glycerol, $1 \mathrm{mM}$ ATP). Purified proteasomes were released with $1 \%$ TEV 


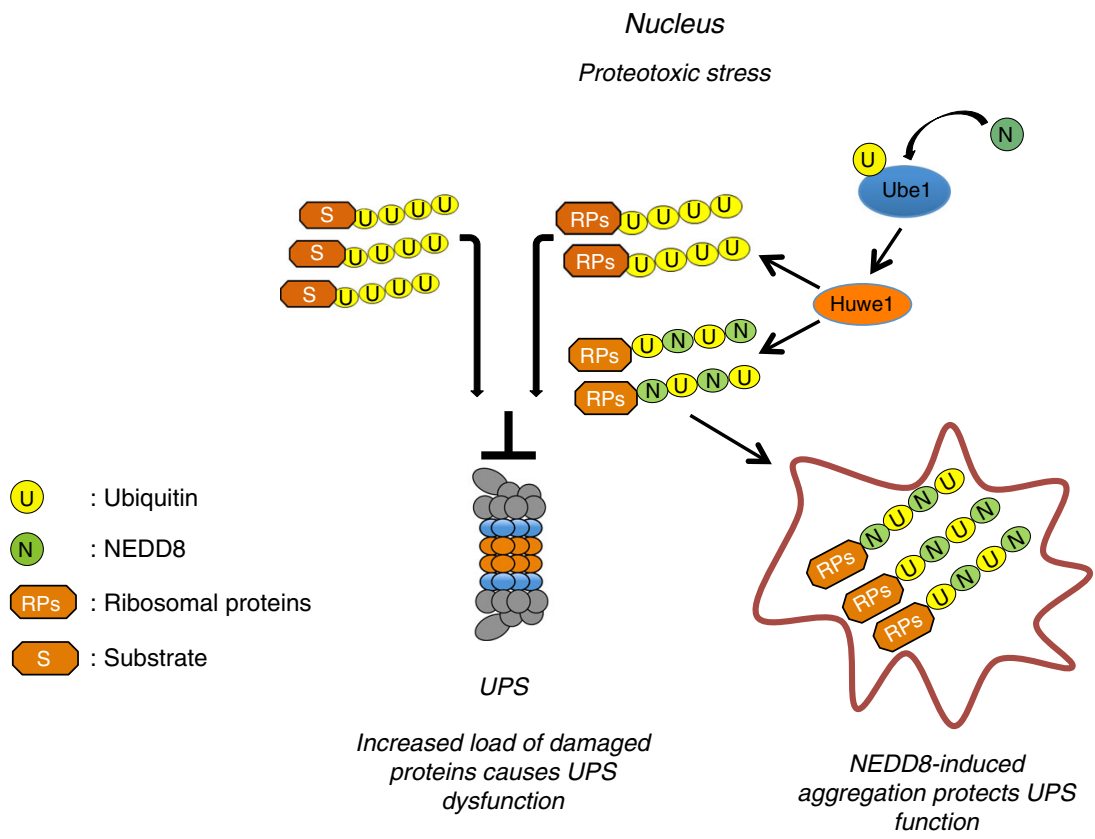

Fig. 10 Model for the role of NEDDylation in nuclear proteotoxic stress response. Upon proteotoxic stress the load of misfolded ubiquitinated proteins is increased compromising the function of UPS. The activation of NEDD8 by the ubiquitin E1 enzyme UBA1 and its conjugation by E3-ligases such as HUWE1 results in the modification of substrates with NEDD8 and ubiquitin possibly with hybrid NEDD8/ubiquitin chains. This provides a regulatory pathway to prevent substrate targeting to the proteasome, promoting the transient aggregation of a group of proteins. This protects the UPS from the stress-induced toxicity

protease at $30^{\circ} \mathrm{C}, 1 \mathrm{~h}$. After measuring protein concentration proteasomes were kept at $-80^{\circ} \mathrm{C}$.

One microgram of purified $26 \mathrm{~S}$ proteasomes was used in an in vitro processing/ degradation assay with $300 \mathrm{ng}$ of purified $\mathrm{His}_{6}$-tagged conjugates in $40 \mu \mathrm{l}$ reactions containing $25 \mathrm{mM}$ Tris- $\mathrm{HCl}, \mathrm{pH} 7.5,10 \mathrm{mM} \mathrm{MgCl}_{2}, 10 \%$ glycerol, $1 \mathrm{mM}$ ATP, 1 $\mathrm{mM}$ DTT at $37^{\circ} \mathrm{C}$ as described in ref. ${ }^{66}$. Reactions were stopped by the addition of $40 \mu \mathrm{l}$ of $2 \times$ SDS Laemmli's buffer and analysed by western blotting.

\section{Data availability}

The SILAC proteomics data have been deposited in the MassIVE repository (https:// massive.ucsd.edu/) with the accession code MSV000082623. All other data supporting the findings of this study are available from the corresponding author on reasonable request.

Received: 11 August 2017 Accepted: 24 August 2018

Published online: 22 October 2018

\section{References}

1. Chen, B., Retzlaff, M., Roos, T. \& Frydman, J. Cellular strategies of protein quality control. Cold Spring Harb. Perspect. Biol. 3, a004374 (2011).

2. Richter, K., Haslbeck, M. \& Buchner, J. The heat shock response: life on the verge of death. Mol. Cell 40, 253-266 (2010).

3. de Oliveira, G. A., Rangel, L. P., Costa, D. C. \& Silva, J. L. Misfolding, aggregation, and disordered segments in c-Abl and p53 in human cancer. Front. Oncol. 5, 97 (2015).

4. Hsu, A. L., Murphy, C. T. \& Kenyon, C. Regulation of aging and age-related disease by DAF-16 and heat-shock factor. Science 300, 1142-1145 (2003)

5. Morimoto, R. I. Proteotoxic stress and inducible chaperone networks in neurodegenerative disease and aging. Genes Dev. 22, 1427-1438 (2008).

6. Taylor, J. P., Hardy, J. \& Fischbeck, K. H. Toxic proteins in neurodegenerative disease. Science 296, 1991-1995 (2002).

7. Valastyan, J. S. \& Lindquist, S. Mechanisms of protein-folding diseases at a glance. Dis. Model Mech. 7, 9-14 (2014).

8. Amm, I., Sommer, T. \& Wolf, D. H. Protein quality control and elimination of protein waste: the role of the ubiquitin-proteasome system. Biochim. Biophys. Acta 1843, 182-196 (2014).

9. Kirkin, V., McEwan, D. G., Novak, I. \& Dikic, I. A role for ubiquitin in selective autophagy. Mol. Cell 34, 259-269 (2009).
10. Metzger, M. B., Hristova, V. A. \& Weissman, A. M. HECT and RING finger families of E3 ubiquitin ligases at a glance. J. Cell Sci. 125, 531-537 (2012).

11. Collins, G. A. \& Goldberg, A. L. The logic of the $26 \mathrm{~S}$ proteasome. Cell 169, 792-806 (2017)

12. Finley, D. Recognition and processing of ubiquitin-protein conjugates by the proteasome. Annu. Rev. Biochem. 78, 477-513 (2009).

13. Savulescu, A. F., Rotem, A. \& Harel, A. Proteasomes crossing the nuclear border. Nucleus 2, 258-263 (2011).

14. Bence, N. F., Sampat, R. M. \& Kopito, R. R. Impairment of the ubiquitinproteasome system by protein aggregation. Science 292, 1552-1555 (2001).

15. Deriziotis, P. et al. Misfolded PrP impairs the UPS by interaction with the $20 \mathrm{~S}$ proteasome and inhibition of substrate entry. EMBO J. 30, 3065-3077 (2011).

16. Lindersson, E. et al. Proteasomal inhibition by alpha-synuclein filaments and oligomers. J. Biol. Chem. 279, 12924-12934 (2004).

17. Lorenzo, A. \& Yankner, B. A. Amyloid fibril toxicity in Alzheimer's disease and diabetes. Ann. N.Y. Acad. Sci. 777, 89-95 (1996).

18. Bennett, E. J., Bence, N. F., Jayakumar, R. \& Kopito, R. R. Global impairment of the ubiquitin-proteasome system by nuclear or cytoplasmic protein aggregates precedes inclusion body formation. Mol. Cell 17, 351-365 (2005).

19. Kim, Y. E. et al. Soluble oligomers of PolyQ-Expanded Huntingtin target a multiplicity of key cellular factors. Mol. Cell 63, 951-964 (2016).

20. Schipper-Krom, S., Juenemann, K. \& Reits, E. A. The ubiquitin-proteasome system in Huntington's disease: are proteasomes impaired, initiators of disease, or coming to the rescue? Biochem. Res. Int. 2012, 837015 (2012).

21. Treusch, S., Cyr, D. M. \& Lindquist, S. Amyloid deposits: protection against toxic protein species? Cell Cycle 8, 1668-1674 (2009).

22. Golebiowski, F. et al. System-wide changes to SUMO modifications in response to heat shock. Sci. Signal. 2, ra24 (2009).

23. Guo, L. et al. A cellular system that degrades misfolded proteins and protects against neurodegeneration. Mol. Cell 55, 15-30 (2014).

24. Abidi, N. \& Xirodimas, D. P. Regulation of cancer-related pathways by protein NEDDylation and strategies for the use of NEDD8 inhibitors in the clinic. Endocr. Relat. Cancer 22, T55-T70 (2015).

25. Enchev, R. I., Schulman, B. A. \& Peter, M. Protein neddylation: beyond cullinRING ligases. Nat. Rev. Mol. Cell Biol. 16, 30-44 (2015).

26. Dil Kuazi, A. et al. NEDD8 protein is involved in ubiquitinated inclusion bodies. J. Pathol. 199, 259-266 (2003).

27. Mori, F. et al. Accumulation of NEDD8 in neuronal and glial inclusions of neurodegenerative disorders. Neuropathol. Appl. Neurobiol. 31, 53-61 (2005).

28. Stenoien, D. L. et al. Polyglutamine-expanded androgen receptors form aggregates that sequester heat shock proteins, proteasome components and 
SRC-1, and are suppressed by the HDJ-2 chaperone. Hum. Mol. Genet. 8, 731-741 (1999).

29. Richet, E. et al. NUB1 modulation of GSK3beta reduces tau aggregation. Hum. Mol. Genet. 21, 5254-5267 (2012).

30. Lu, B. et al. Identification of NUB1 as a suppressor of mutant Huntington toxicity via enhanced protein clearance. Nat. Neurosci. 16, 562-570 (2013).

31. Schulman, B. A. \& Harper, J. W. Ubiquitin-like protein activation by E1 enzymes: the apex for downstream signalling pathways. Nat. Rev. Mol. Cell Biol. 10, 319-331 (2009).

32. Leidecker, O., Matic, I., Mahata, B., Pion, E. \& Xirodimas, D. P. The ubiquitin E1 enzyme Ube1 mediates NEDD8 activation under diverse stress conditions. Cell Cycle 11, 1142-1150 (2012).

33. Xu, G. et al. Vulnerability of newly synthesized proteins to proteostasis stress. J. Cell Sci. 129, 1892-1901 (2016).

34. Castoralova, M. et al. SUMO-2/3 conjugates accumulating under heat shock or MG132 treatment result largely from new protein synthesis. Biochim. Biophys. Acta 1823, 911-919 (2012).

35. Hipp, M. S., Park, S. H. \& Hartl, F. U. Proteostasis impairment in proteinmisfolding and -aggregation diseases. Trends Cell Biol. 24, 506-514 (2014).

36. Johnston, J. A., Ward, C. L. \& Kopito, R. R. Aggresomes: a cellular response to misfolded proteins. J. Cell Biol. 143, 1883-1898 (1998).

37. Enserink, J. M. Sumo and the cellular stress response. Cell Div. 10, 4 (2015).

38. Hendriks, I. A. \& Vertegaal, A. C. A comprehensive compilation of SUMO proteomics. Nat. Rev. Mol. Cell Biol. 17, 581-595 (2016).

39. Sundqvist, A., Liu, G., Mirsaliotis, A. \& Xirodimas, D. P. Regulation of nucleolar signalling to p53 through NEDDylation of L11. EMBO Rep. 10 1132-1139 (2009).

40. Bence, N. F., Bennett, E. J. \& Kopito, R. R. Application and analysis of the GFPu family of ubiquitin-proteasome system reporters. Methods Enzymol. 399, 481-490 (2005).

41. Xirodimas, D. P. et al. Ribosomal proteins are targets for the NEDD8 pathway. EMBO Rep. 9, 280-286 (2008).

42. Sung, M. K., Reitsma, J. M., Sweredoski, M. J., Hess, S. \& Deshaies, R. J. Ribosomal proteins produced in excess are degraded by the ubiquitinproteasome system. Mol. Biol. Cell 27, 2642-2652 (2016).

43. Hjerpe, R. et al. Changes in the ratio of free NEDD8 to ubiquitin triggers NEDDylation by ubiquitin enzymes. Biochem. J. 441, 927-936 (2012).

44. Li, J. et al. NEDD8 ultimate buster 1 long (NUB1L) protein suppresses atypical Neddylation and promotes the proteasomal degradation of misfolded proteins. J. Biol. Chem. 290, 23850-23862 (2015).

45. Liu, G. \& Xirodimas, D. P. NUB1 promotes cytoplasmic localization of p53 through cooperation of the NEDD8 and ubiquitin pathways. Oncogene 29, 2252-2261 (2010).

46. Sung, M. K. et al. A conserved quality-control pathway that mediates degradation of unassembled ribosomal proteins. Elife 5, https://doi.org/ 10.7554/eLife.19105 (2016)

47. Wang, X. et al. Mass spectrometric characterization of the affinity-purified human 26S proteasome complex. Biochemistry 46, 3553-3565 (2007).

48. Park, S. H. et al. PolyQ proteins interfere with nuclear degradation of cytosolic proteins by sequestering the Sis1p chaperone. Cell 154, 134-145 (2013).

49. Prasad, R., Kawaguchi, S. \& Ng, D. T. A nucleus-based quality control mechanism for cytosolic proteins. Mol. Biol. Cell 21, 2117-2127 (2010).

50. Russell, S. J., Steger, K. A. \& Johnston, S. A. Subcellular localization, stoichiometry, and protein levels of $26 \mathrm{~S}$ proteasome subunits in yeast. J. Biol. Chem. 274, 21943-21952 (1999).

51. Lam, Y. W., Lamond, A. I., Mann, M. \& Andersen, J. S. Analysis of nucleolar protein dynamics reveals the nuclear degradation of ribosomal proteins. Curr. Biol. 17, 749-760 (2007).

52. Latonen, L., Moore, H. M., Bai, B., Jaamaa, S. \& Laiho, M. Proteasome inhibitors induce nucleolar aggregation of proteasome target proteins and polyadenylated RNA by altering ubiquitin availability. Oncogene 30, 790-805 (2011).

53. Miller, S. B. et al. Compartment-specific aggregases direct distinct nuclear and cytoplasmic aggregate deposition. EMBO J. 34, 778-797 (2015).

54. Singh, R. K. et al. Recognition and cleavage of related to ubiquitin 1 (Rub1) and Rub1-ubiquitin chains by components of the ubiquitin-proteasome system. Mol. Cell. Proteom. 11, 1595-1611 (2012).

55. David, D. C. et al. Widespread protein aggregation as an inherent part of aging in C. elegans. PLoS Biol. 8, el000450 (2010).

56. Walther, D. M. et al. Widespread proteome remodeling and aggregation in aging C. elegans. Cell 161, 919-932 (2015).

57. Arrasate, M., Mitra, S., Schweitzer, E. S., Segal, M. R. \& Finkbeiner, S. Inclusion body formation reduces levels of mutant huntingtin and the risk of neuronal death. Nature 431, 805-810 (2004).
58. Ferreira, S. T., Vieira, M. N. \& De Felice, F. G. Soluble protein oligomers as emerging toxins in Alzheimer's and other amyloid diseases. IUBMB Life 59, 332-345 (2007)

59. Tatham, M. H., Rodriguez, M. S., Xirodimas, D. P. \& Hay, R. T. Detection of protein SUMOylation in vivo. Nat. Protoc. 4, 1363-1371 (2009).

60. Xirodimas, D., Saville, M. K., Edling, C., Lane, D. P. \& Lain, S. Different effects of p14ARF on the levels of ubiquitinated p53 and Mdm2 in vivo. Oncogene $\mathbf{2 0}$, 4972-4983 (2001).

61. Bailly, A. et al. The NEDD8 inhibitor MLN4924 increases the size of the nucleolus and activates p53 through the ribosomal-Mdm2 pathway. Oncogene 35, 415-426 (2016).

62. Bolte, S. \& Cordelieres, F. P. A guided tour into subcellular colocalization analysis in light microscopy. J. Microsc. 224, 213-232 (2006).

63. Sun, W. et al. Microwave-assisted protein preparation and enzymatic digestion in proteomics. Mol. Cell Proteom. 5, 769-776 (2006).

64. Mahata, B., Sundqvist, A. \& Xirodimas, D. P. Recruitment of RPL11 at promoter sites of p53-regulated genes upon nucleolar stress through NEDD8 and in an Mdm2-dependent manner. Oncogene 31, 3060-3071 (2012).

65. Guo, X. et al. UBLCP1 is a $26 \mathrm{~S}$ proteasome phosphatase that regulates nuclear proteasome activity. Proc. Natl Acad. Sci. USA 108, 18649-18654 (2011).

66. Chojnacki, M. et al. Polyubiquitin-photoactivatable crosslinking reagents for mapping ubiquitin interactome identify Rpn1 as a proteasome ubiquitinassociating subunit. Cell Chem. Biol. 24, 443-457 e446 (2017).

\section{Acknowledgements}

We are grateful to Olivier Coux for his assistance in the proteasome activity assays, the Montpellier Rio Imaging (MRI) facility for the microscopy, Prof. Ron Kopito and Dr. Lan Huang for cell lines. The study was financially supported by the Labex EpiGenMed, an "Investissements d'avenir" programme, reference ANR-10-LABX-12-01, INCa (PLBIO16-251), the ATIP/AVENIR programme, INSERM and the COST Action (PROTEOSTASIS BM1307-European Cooperation in Science and Technology).

\section{Author contributions}

C.M.M. with the assistance of H.T. performed the majority of the experiments with the exception of the proteomic studies, which were performed by S.L.-G. and the confocal microscopy performed by A.P., S.U. and P.M. performed the proteomic analysis. D.P.X., C.M.M. and S.L.G. designed the experiments with the assistance of M.S.R., D.P.X. supervised the study, wrote the manuscript with the help of all co-authors.

\section{Additional information}

Supplementary Information accompanies this paper at https://doi.org/10.1038/s41467018-06365-0.

Competing interests: The authors declare no competing interests

Reprints and permission information is available online at http://npg.nature.com/ reprintsandpermissions/

Publisher's note: Springer Nature remains neutral with regard to jurisdictional claims in published maps and institutional affiliations.

Open Access This article is licensed under a Creative Commons Attribution 4.0 International License, which permits use, sharing, adaptation, distribution and reproduction in any medium or format, as long as you give appropriate credit to the original author(s) and the source, provide a link to the Creative Commons license, and indicate if changes were made. The images or other third party material in this article are included in the article's Creative Commons license, unless indicated otherwise in a credit line to the material. If material is not included in the article's Creative Commons license and your intended use is not permitted by statutory regulation or exceeds the permitted use, you will need to obtain permission directly from the copyright holder. To view a copy of this license, visit http://creativecommons.org/ licenses/by/4.0/.

(c) The Author(s) 2018 